\title{
The Monaural Nuclei of the Lateral Lemniscus in an Echolocating Bat: Parallel Pathways for Analyzing Temporal Features of Sound
}

\author{
Ellen Covey and John H. Casseday \\ Department of Neurobiology, Duke University Medical Center, Durham, North Carolina 27710
}

In echolocating bats, three cell groups in the lateral lemniscus are conspicuous for their large size and high degree of differentiation. These cell groups are the intermediate nucleus (INLL), columnar nucleus (VNLLC), and multipolar cell area (VNLLm). All receive projections from the contralateral cochlear nucleus. Previous anatomical studies suggest the hypothesis that these nuclei are important for analyzing the temporal structure of sound. To investigate this possibility, we recorded responses of single units in the INLL, VNLLC, and VNLLm of Eptesicus fuscus. The results show that each cytoarchitectural division contains a complete tonotopic representation. Certain response properties are common to all three nuclei. First, virtually all units are monaural. Second, all are broadly tuned to frequency; their average $\boldsymbol{Q}_{\text {10dB }}$ value of 9.1 is considerably lower than values measured in the inferior colliculus of Eptesicus. Third, most units have little or no spontaneous activity. Fourth, all have short integration times, responding robustly to stimuli less than $5 \mathrm{msec}$ in duration. The broad tuning, lack of spontaneous activity, and short integration time all make these neurons well suited for the accurate encoding of temporal information. Although there are many similarities, there are also important differences among nuclei. The clearest evidence of specialization is in VNLLc. Neurons here are more broadly tuned than those in INLL or VNLLm, have no spontaneous activity, and always respond with one spike per stimulus. The latency of the spike is precisely locked to the stimulus onset, with variability from trial to trial as low as $\mathbf{0 . 0 3}$ msec. In addition, the latency remains constant over large variations in frequency or intensity. In INLL and VNLLm, response patterns are about equally distributed between tonic, chopping, and phasic; there are no single-spike constant-latency responses of the type seen in VNLLc, although some choppers and pausers do respond with constant first-spike latency. The results indicate that VNLLc is specialized to encode very precisely the onset of sound; the other nuclei may encode ongoing properties of a sound.

In echolocating bats and dolphins, one set of cell groups that lies within the lateral lemniscus is unusually orderly in structure and greatly expanded in size (Poljak, 1926; Zook and Casseday,

Received Mar. 8, 1991; revised June 5, 1991; accepted June 19, 1991

This research was supported by NIH Grants DC-607 and DC-287. We thank Yan Liu and Boma Rosemond for technical assistance, and John Whitehead for his help in preparing the illustrations. We also thank Drs. C. Tsuchitani, J. J. Blum, J. A. Simmons, and L. Kitzes for their critical reading of the manuscript and many helpful comments.

Correspondence should be addressed to Ellen Covey, Department of Neurobiology, Box 3209, Duke University Medical Center, Durham, NC 27710. Copyright (C) 1991 Society for Neuroscience $0270-6474 / 91 / 113456-15 \$ 05.00 / 0$ 1982a; Zook et al., 1988). This high degree of development and differentiation in two very different mammalian orders suggests that these nuclei play an important role in echolocation. In a previous study on the tonotopically organized connections of this area, we suggested that their function might be to analyze and encode information about the temporal structure of sequential auditory signals such as pulse-echo pairs (Covey and Casseday, 1986). The present study explores this pussibility using single-unit methods to describe the response to sound of cells in the lateral lemniscal nuclei of the big brown bat.

The hypertrophied cell groups are those that lie below the dorsal nucleus of the lateral lemniscus (DNLL). These are the intermediate nucleus (INLL) and two parts of the ventral nucleus, the columnar area (VNLLc) and the multipolar cell area (VNLLm). In this report, we shall restrict our observations to the hypertrophied parts. There is ample justification for treating these parts separately from DNLL. The carlicr connectional studies drew attention to an important functional distinction between DNII, and the cell groups ventral to it. The DNLL receives projections from the superior olivary complex and thus is connected with the binaural system (e.g., Glendenning et al., 1981; Zook and Casseday, 1982b). As would be suggested by its connections, the response properties of neurons in DNLL in the cat are binaural (Aitkin et al., 1970; Brugge et al., 1970). In contrast, INLL, VNLLc, and VNLLm appear to be monaural. That is, they receive input mainly from the contralateral cochlear nucleus (e.g., Glendenning et al., 1981; Zook and Casseday, 1982b; Covey and Casseday, 1986). This observation raises the idea that the INLL, VNLLc, and VNLLm are components of a system that functions monaurally, in parallel to the binaural system. If so, one would expect that neurons within the entire complex of cell groups ventral to DNLL would have some response properties in common, at lcast that of monaural response to sound. There is evidence that, in the cat, most neurons ventral to DNLI are monaural (Aitkin et al., 1970). For convenience, we shall refer to the INLL, VNLLc, and VNLLm collectively as the "monaural nuclei" or "monaural complex" of the lateral lemniscus.

Previous anatomical evidence also suggests that, although the monaural nuclei may perform related functions, each one represents a separate parallel pathway from the cochlear nucleus to the midbrain. Studies of anterograde transport show that pathways from the anteroventral cochlear nucleus (AVCN) diverge to each nucleus, and studies of retrograde transport show that each structure sends a separate projection to the inferior colliculus (Zook and Casseday, 1982b; Covey and Casseday, 1986). The idea of separate pathways is supported by electrophysiological evidence of repeated tonotopic representations in the lateral lemniscus of the horseshoe bat (Metzner and RadtkcSchuller, 1987). 
Among the monaural pathways, the VNLLc is especially intriguing. It consists of a three-dimensional matrix of cells in which the projections from the $\Lambda \mathrm{VCN}$ converge onto sheets of cells; each projection sheet is one cell thick. The cells within the sheets are innervated by calyceal endings. These observations led to the idea that temporal processing might be important in the VNLLc (Covey and Casseday, 1986).

Because of these structural hints, we looked for functional evidence of parallel processing and specialization for encoding temporal information. We recorded responses to sound from single units in the monaural complex of the big brown bat, Eptesicus fuscus. Our aims were, first, simply to characterize the basic response properties of the neurons. We were interested in whether the complex as a whole has response properties that are common to the system, and whether these response properties would provide clues about what this common function is. Second, to test the idea that the monaural complex consists of parallel pathways, we looked for separate tonotopic representations in cach cytoarchitectural and connectional subdivision. Finally, we asked whether there were differences in the response properties of neurons in the different divisions.

\section{Materials and Methods}

Surgical procedures. The animals used in this study were 21 big brown bats (Eptesicus fuscus) of both sexes, obtained from the attics of local houses. On the day prior to recording, the bat was anesthetized with a combination of Metofane (methoxyflurane) and Innovar-Vet (fentanyl, $0.4 \mathrm{mg} / \mathrm{ml}$, plus droperidol, $20 \mathrm{mg} / \mathrm{ml} ; 0.125 \mathrm{mg} / \mathrm{kg}$ ). Its head was held in a specially designed bite-bar that was attached to manipulators that allowed the head to be rotated in three dimensions. Fine adjustments were made in the orientation of the skull so that it conformed to a standard stereotaxic position. Then, a metal post was attached to the skull with cyanoacrylate adhesive and dental cement. The post was constructed so that the placement of the bat in the stereotaxic apparatus (Kopf, modified for bats) could be replicated precisely from one day's recording session to the next. Each bat was used in one to six recording sessions, on separate days. Each session was $6 \mathrm{hr}$ or less in duration. Between recording sessions, bats were housed in individual cages and given free access to food and water. The cages were located in a temperature and humidity controlled room.

Recording began on the day following implantation of the post. Prior to placement in the stereotaxic device, the bat was tranquilized with Innovar-Vet and lightly anesthetized with Metofane. A small opening, less than $1 \mathrm{~mm}$ in diameter, was made in the skull overlying the rostrolateral part of the inferior colliculus. Between recording sessions, the opening was covered with a coat of Vaseline. The animal was allowed to recover from the anesthesia for at least $30 \mathrm{~min}$ before recording began. This recovery period was sufficient for stability of neural responses. During recording, local anesthetic was applied to the scalp incision. During the recording sessions, the bat was restrained in a foam-lined holder that was molded to the shape of the body so as to hold it firmly but comfortably. The holder was suspended in an elastic sling to damp movements. The absence of struggling or excessive vocalization indicated that there was no undue pressure or injury to the bat.

Acoustic stimulation and recording. The auditory stimuli used were pure tones, frequency-modulated sweeps (FM), or noise bursts. The stimuli were usually $5 \mathrm{msec}$ in duration, with a $1 \mathrm{msec}$ rise-fall time, presented at a rate of $3 / \mathrm{sec}$. For specific experiments, stimulus parameters were varied as described in the Results. Stimuli were presented binaurally via two Bruel and $\mathrm{Kjaer} 1 / 4^{\prime \prime}$ condenser microphones modified for use as loudspeakers and placed as close as possible to the external ear, at a distance of $1 \mathrm{~mm}$ or less. The output of the loudspeakers was measured with a $1 / 8^{\prime \prime}$ Bruel and Kjaer microphone and was found to be flat $\pm 5 \mathrm{~dB}$ between 20 and $100 \mathrm{kHz}$. Using these measurements, we converted the sound levels used during the experiments from attenuator settings to sound pressure levels (SPL re $20 \mu \mathrm{Pa}$ ). Cross talk between the two ears was measured by presenting sound at one ear and measuring the sound at that ear and at the opposite ear. The results indicated that, over the frequency range we used, the SPL at the ear opposite the source was at least $30 \mathrm{~dB}$ below that at the ear next to the source.
Neural response properties were mapped with glass micropipelte electrodes filled with $2 \mathrm{M} \mathrm{KCl}$ or a $5 \%$ solution of HRP (Sigma) in physiological saline. The micropipettes had tip diameters of $<1.0 \mu \mathrm{m}$ and impedances ranging from 10 to $40 \mathrm{M} \Omega$. The electrode was aimed stereotaxically and advanced using a Kopf stepping hydraulic microdrive. Because of the high cell-packing density in the lateral lemniscal nuclei of the bat, the possibility of recording from fibers was less than it would be in many other mammalian species. Nevertheless, we only collected data from units that we could be confident were cell bodies, based on the size and waveform of their action potentials. In addition, such units could be "killed" by advancing the electrode, in which case they fired at a very high rate beforc bccoming silent. Frequently, prepotentials were seen in recordings from units that met these criteria.

Data were collected on an IBM PC-AT computer and accessory hardware and software (Modular Instruments) for data collection, storage, and creation of poststimulus time histograms (PSTHs) and dot raster displays. The data were collected using a bin width of $1.0 \mu \mathrm{sec}$. Further statistical analysis of first spike latencies, interspike intervals (ISIs), chopping rates, and so on, was performed using computer spreadsheets (LoTUs 1-2-3).

Whenever a unit was isolated, certain routine tests were conducted. First, we determined the unit's best frequency (BF), that is, the frequency at which threshold was lowest, and obtained a tuning curve in $10 \mathrm{~dB}$ steps. From the tuning curve, we derived the $Q_{10 \mathrm{~dB}}(\mathrm{BF}$ divided by the bandwidth of the tuning curve $10 \mathrm{~dB}$ above threshold). To test for binaural responsiveness, stimuli were first presented to cach car separately and then to both ears simultaneously, varying interaural sound level difference. Data sets for PSTHs and dot raster displays were then obtained at the unit's BF. Each data set consisted of the responses to 100 stimulus presentations under constant conditions. Data sets were obtained in $10 \mathrm{~dB}$ steps, starting at $10 \mathrm{~dB}$ above the unit's minimum threshold. From these data, we could determine spontaneous discharge rate, sound-evoked discharge rate, latency of the first spike, latency of subsequent spikes, variability of first-spike latency, interspike interval, and distribution of spikes over time. In addition, we obtained rate-level functions and binaural interaction functions, if applicable.

Finally, special tests were conducted, depending on the unit's response characteristics. These included varying stimulus duration, interstimulus interval, or repetition rate, or testing sensitivity to FM stimuli.

Histological procedures. Multiple electrode penetrations were made in each animal, and selected recording sites were marked with small iontophoretic injections of HRP. These injections marked the locations of neurons with specific response properties and served as reference points in the reconstruction of electrode penetrations. One to four injections were made in a single animal, at dorsal-ventral levels separated by at least $400 \mu \mathrm{m}$. The injections were made using a pulsed ( $7 \mathrm{sec}$ on, $7 \mathrm{sec}$ off) current of $0.75-0.9 \mu \mathrm{A}$, positive at the electrode, applied for 2.0-3.5 min.

After the final recording session, animals were administered a lethal dose of Nembutal (pentobarbital) and perfused through the heart with phosphate-buffered saline followed by a fixation solution of $4 \%$ glutaraldehyde in phosphate buffer. The brain was removed and refrigerated overnight in a solution of $30 \%$ sucrose in phosphate buffer. Sections 40 $\mu \mathrm{m}$ thick were cut on a freezing microtome and processed with tetramethylbenzidine (TMB) according to the method of Mesulam (1978). Sections were first dehydrated and coverslipped without staining, and injection sites and transport were plotted. The sections were then decoverslipped and stained with cresyl violet to examine cytoarchitecture.

As a supplement to previous cytoarchitectural studies (Zook and Casseday, 1982a; Covey and Casseday, 1986), Golgi-impregnated sections were prepared using a modification (Adams, 1979b) of the GolgiKopsch technique (Stensaas and Stensaas, 1968). Golgi-impregnated neurons were drawn using a camera lucida and $100 \times$ oil-immersion objective.

\section{Results}

Cytoarchitecture: three divisions with unique organization and cell types

In Eptesicus fuscus, the region ventral to DNLL can clearly be divided into three major cytoarchitectonic areas: the INLL, the VNLLc, and the VNLLm. All these nuclei are bounded on the medial and lateral sides by the ascending fibers of the lateral 


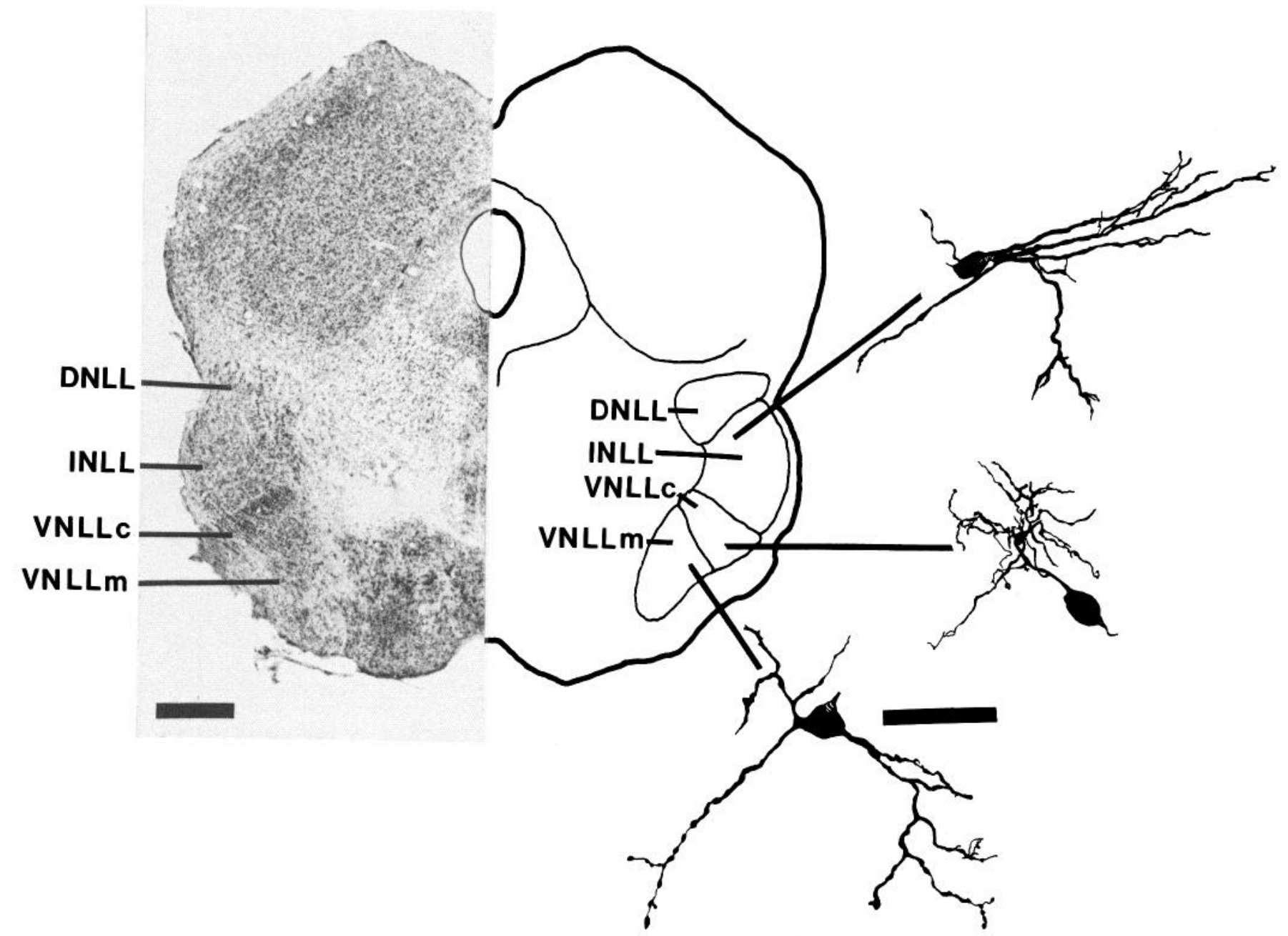

Figure 1. Examples of the location, size, and appearance of the main cell types in the $I N L L, V N L L c$, and $V N L L m$. On the left is a low-power photomicrograph of a Nissl-stained frontal section through the brainstem. On the right is a drawing to show the cytoarchitectural divisions and camera lucida drawings of Golgi-impregnated neurons in the different cell groups. Scale bars: left, $500 \mu \mathrm{m} ;$ right, $50 \mu \mathrm{m}$.

lemniscus. Those fibers that terminate in the nuclei enter at right angles to the ascending fibers (Covey and Casseday, 1986).

Figure 1 illustrates the appearance of Golgi-impregnated cells in each division; these morphological data provide additional evidence that each area contains a distinctive cell population. The INLL contains predominantly elongate neurons with several long dendrites extending from either end of the soma in a plane orthogonal to the ascending fibers of the lateral lemniscus and parallel to the fibers that enter the nucleus. In the INLL cell illustrated in Figure 1, one dendrite is oriented parallel to the ascending fibers.

In the VNLLc, virtually every neuron is of a single type. They are very similar to spherical bushy cells in the anteroventral cochlear nucleus in that they are round to oval in shape and have one large, thick dendrite that branches profusely some distance away from the cell body. At least some of these branches appear to extend parallel to the ascending fibers. The cells are tightly packed in a columnar arrangement between bundles of fibers, hence the name "columnar nucleus" (Covey and Casseday, 1986).

The VNLLm derives its name from the fact that most neurons in this region are multipolar in shape. They are larger than
VNLLc neurons and have several sparsely branching thick dendrites. The cell bodies and dendrites of neurons in VNLLm have no consistent orientation relative to the fibers of the lateral lemniscus.

Tests with binaural stimuli: neurons in INLL, VNLLc, and VNLLm are monaural

We recorded responses from a total of 197 single units and 88 multiunit clusters that could be reliably localized within the lateral lemniscal nuclei. Except for tonotopic mapping, all analyses are based on single units only. Of the total sample, 39 multiand 73 single units were in DNLL, 20 multi- and 68 single units were in INLL, 8 multi- and 18 single units were in VNLLc, and 11 multi- and 17 single units were in VNLLm. Another 8 multiand 16 single units were located in the paralemniscal region medial to the INLL and VNLLc. The responses of the binaural units in DNLL and the paralemniscal area will be described in detail in a separate report.

Figure 2 shows two examples of electrode penetrations through the nuclei of the lateral lemniscus. In DNLL (Fig. 2A), all units encountered were binaural, excited by sound at the contralateral ear, and inhibited by sound at the ipsilateral ear (IE). Through 

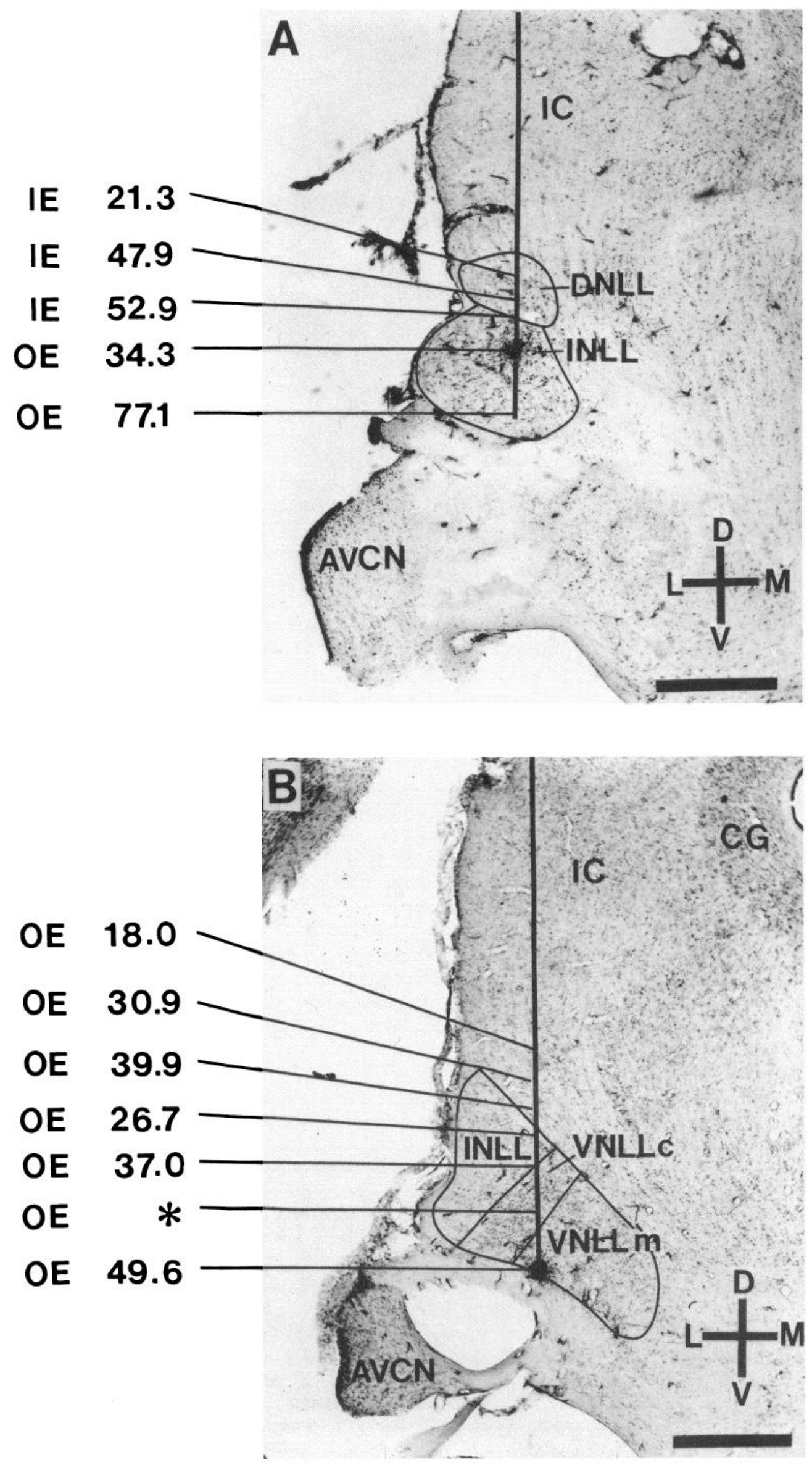

Figure 2. Photomicrographs to illustrate representative electrode penetrations through the lateral lemniscal nuclei and HRP injections used to mark selected recording sites. In all cases, injection sites were typically $50-100 \mu \mathrm{m}$ in diameter, allowing precise localization of the recording sites and penetrations. Both sections are in the frontal plane; the tissue is reacted with TMB and stained with cresyl violet. For each recording site along the penetrations illustrated, the neuron's $\mathrm{BF}$ in $\mathrm{kHz}$ and binaural response type $(I E, O E)$ are marked. In $A$, the electrode passed through the dorsal nucleus of the lateral lemniscus $(D N L L)$ where binaural $(I E)$ neurons were encountered; BFs increased from dorsal to ventral. Upon entering $I N L L$, neurons were monaural $(O E)$, and the sequence of BFs started over again. In $B$, the electrode passed through the area rostral to DNLL, through $I N L L, V N L L C$, and $V N L L m$. For the neuron marked with an asterisk, the tuning curve was too broad near threshold to determine a single BF. All neurons in this penetration were monaural $(O E) . I C$, inferior colliculus; $C G$, central gray. Scale bars, $500 \mu \mathrm{m}$. 


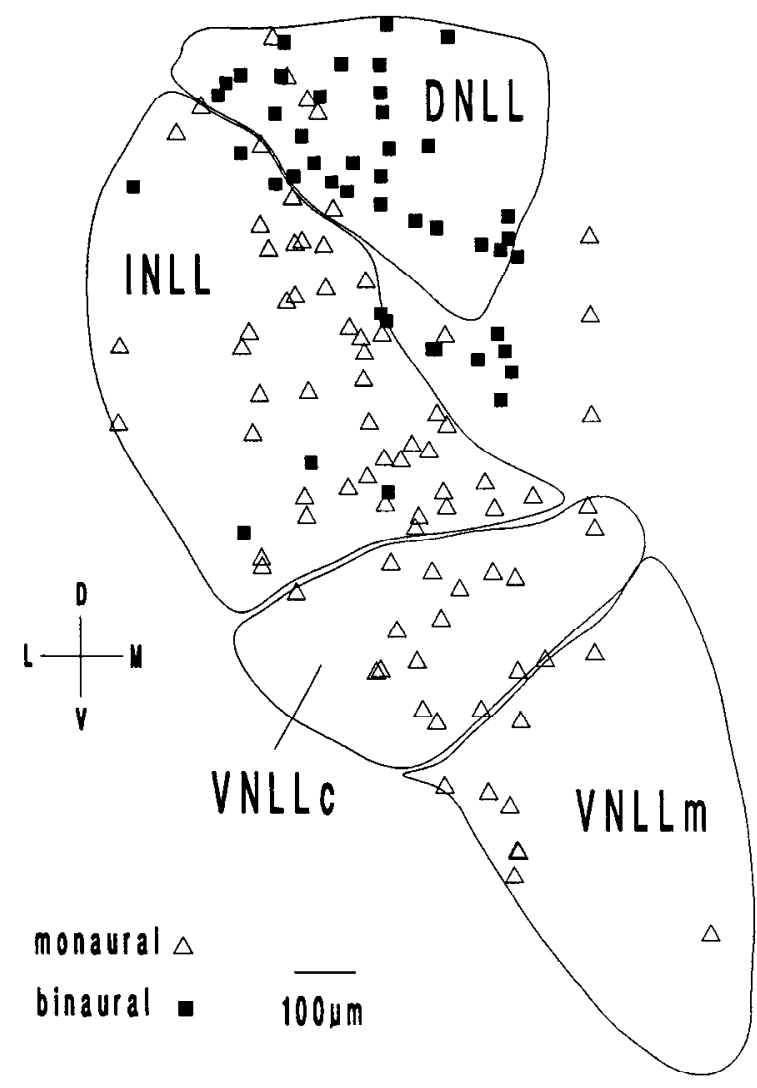

Figure 3. Composite drawing to illustrate the location of monaural and binaural cells in the nuclei of the lateral lemniscus. Data from all experimental animals were pooled and projected on to a single representative section. There is a clear separation into binaural regions $(D N L L$ and the region medial to $I N L L$ and $V N L L C$ ) and monaural regions $(I N L L, V N L L C$, and $V N L L m)$.

DNLL, there was a progression from low to high frequencies. Below the ventral border of DNLL (Fig. $2 A$ ), all units were monaural, excited by sound at the contralateral ear, and unaffected by sound at the ipsilateral ear (OE). At the same point at which the monaural (OE) units were encountered, the frequency progression began again and BFs increased from low to high. Figure $2 B$ shows a section rostral to DNLL. At this level, all neurons, including those dorsal to the INLL, are monaural.

Figure 3 shows a composite section on which data from all cases have been pooled. As would be expected from the anatomical connections, nearly all neurons in INLL, VNLLc, and VNLLm are monaural. All of these monaural neurons were excited by a stimulus at the contralateral ear; ipsilateral stimuli had no measurable effect, either excitatory or inhibitory. Although the sparse sampling of single units in the medial part of VNLLm does not permit the conclusion that there are no binaural cells here, the multiunit data we have from this region suggest that this is the case. Almost all neurons in DNLL were binaural, excited by sound at the contralateral ear, and inhibited by sound at the ipsilateral ear. In addition, a population of binaural auditory neurons were found in the region just medial to INLL and VNLLc. A few binaural units were found in INLL; all but three of these were located at the dorsal or medial borders. Because of the predominance of monaural responses in INLL, VNLLc, and VNLLm, we refer to these nuclei collectively as the monaural nuclei of the lateral lemniscus.

\section{Tonotopy: multiple tonotopic maps and expanded frequency representations}

If each cytoarchitectural division is functionally separate from the others, we would expect to find separate tonotopic representations in each part. The electrode penetrations shown in Figures 2 and 4 support the idea that in each nucleus the complete range of audible frequencies is represented. In VNLLc, the tonotopic map is arranged in a straightforward progression from low frequencies at the dorsal border to high frequencies at the ventral border. In penetrations where BFs were obtained on either side of the VNLLc border, reversals were often seen. In INLL and VNLLm, the tonotopic arrangement did not appear to be as regular. In INLL, low frequencies are clearly represented in the dorsal and lateral part; however, the highest frequencies seem to be in the central core of the nucleus, surrounded by the middle frequencies. In VNLLm, the general organization appears to be low frequencies medial and high frequencies lateral. However, the low and middle frequencies may wrap around to the lateral edge. Our estimate of the tonotopic organization for these three nuclei is shown in Figure $4 D$.

The distribution of BFs was approximately the same in all three monaural nuclei of the lateral lemniscus. In each division, BFs were distributed throughout the audible range, with a broad peak around $28 \mathrm{kHz}$ (Fig. $5 \mathrm{~A}$ ). Anatomical data on projections from the cochlear nucleus to VNLLc show that the target of the frequency range $25-50 \mathrm{kHz}$ is greatly expanded (Covey and Casseday, 1986). This corresponds to the frequency range that is most often present in the main harmonic of Eptesicus's echolocation call (Simmons, 1989). Minimum threshold as a function of BF is illustrated in Figure $5 B$. Across the entire sample of neurons, $B F$ thresholds varied from about $0 \mathrm{~dB}$ SPL to almost $80 \mathrm{~dB}$ SPL. Units with BFs falling in the range of the expanded frequency representation had the lowest thresholds. There were no systematic differences in BF threshold among neurons located in INLL or VNLLm. In both nuclei, BF thresholds ranged from 2 to about $70 \mathrm{~dB}$ SPL, with mean values of 40.3 and 41.3 dB SPL, respectively. Neurons in VNLLc generally had higher $\mathrm{BF}$ thresholds, ranging from 30 to $78 \mathrm{~dB}$ SPL, with a mean of 53.4 dB SPL.

In addition to responding to pure tones, virtually all units in INLL, VNLLc, and VNLLm responded reliably to noise and to FM sweeps passing through their BF. Their thresholds for these stimuli were similar to those for pure tones at BF.

\section{Frequency tuning curves: VNLLc neurons are broadly tuned}

It is important for the later examination of temporal processing to determine the frequency-filtering characteristics of neurons in the monaural lateral lemniscal nuclei. Convergence of multiple channels in the frequency domain can provide a high level of precision in the temporal domain. However, the additional accuracy in temporal processing is gained at the expense of frequency acuity. Comparison of tuning curves from units in INLL, VNLLc, and VNLLm showed that those in VNLLc (Fig. $6 B$ ) were more broadly tuned than were units in the other monaural nuclei of the lateral lemniscus. VNLLc neurons typically had asymmetrical tuning curves that broadened rapidly above threshold and resembled those of broad-band filters with a steep upper cutoff and a very shallow lower cutoff in the range below $\mathrm{BF}$. In fact, in the range below $\mathrm{BF}$, the tuning curve was virtually flat a few decibels above BF threshold. It was only because these neurons had no spontaneous activity that we were able to de- 


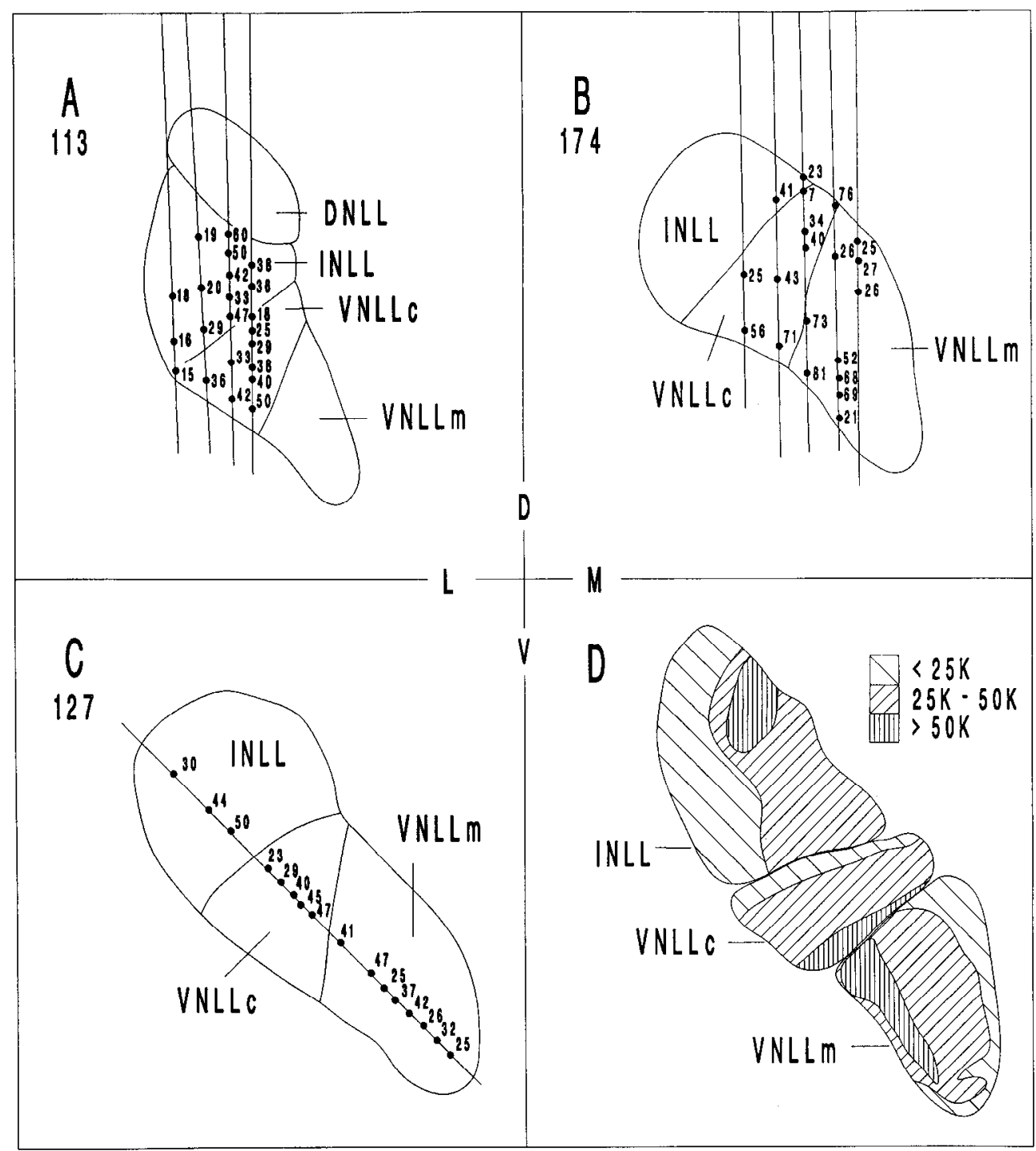

Figure 4. $A, B$, and $C$ illustrate individual electrode penetrations in three different cases. Case numbers are given at the upper left in $A-C$. In each case, the tonotopic progrcssion can be seen within each cytoarchitectural division. $D$ is a schematic drawing to show our estimate of the tonotopic organization of the monaural nuclei of the lateral lemniscus. It is based on a composite reconstruction containing BFs from single units in 21 animals that were pooled and projected onto a single representative section. Although the rostrocaudal dimension is collapsed in this reconstruction, it provides a good approximation of the tonotopic axis in the dorsoventral and mediolateral dimensions. termine a BF at just a few decibels below the broad response area. Neurons in INLL (Fig. 6A) and VNLLm (Fig. 6C) typically had symmetrical V-shaped tuning curves with similar upper and lower slopes. These tuning curves broadened gradually and progressively as sound level increased. The tuning characteristics of each cell group are reflected in their $Q_{10 \mathrm{~dB}}$ values, as shown in Figure 7. The $Q_{10 \mathrm{~dB}}$ values for VNLLc neurons were between 1.3 and 11.2 (mean, 5.1); those for INLL neurons ranged from 2.6 to 27.3 (mean, 11.6), and those for VNLLm neurons were between 3.4 and 33.2 (mean, 8.2). Although there are differences between the $Q_{10 \mathrm{~dB}}$ values for neurons in different monaural nuclei of the lateral lemniscus, the average $Q_{10 \mathrm{~dB}}$ value of 9.1 for all neurons in the monaural regions of the lateral lemniscus is considerably lower than the average $Q_{10 \mathrm{~dB}}$ value of 17.7 obtained from a sample of 123 single units in the central nucleus of the inferior colliculus of Eptesicus (J. H. Casseday and E. Covey, unpublished observations). Thus, it appears that, on the average, neurons in all three monaural cell groups of the lateral lemniscus are more broadly tuned than neurons at the level of the midbrain. The cells in VNLLc are especially imprecise frequency encoders.

\section{Discharge patterns: classes of neurons suited for encoding} temporal information

A common characteristic of all neurons in the monaural nuclei of the lateral lemniscus is that they have little or no spontaneous activity. However, on the basis of the discharge pattern evoked by pure tone bursts, they can be classified into distinct populations. In most cases, it was possible to apply the same response classes that have been used to describe neurons in the cochlear nucleus (Pfeiffer, 1966). Our classifications were based on responses to pure tones at the unit's BF, $20 \mathrm{~dB}$ above threshold. Figure 8 shows examples of the response classes, and Figure 9 shows the distribution of the different response classes in INLL, VNLLc, and VNLLm.

"Phasic" neurons (Fig. 8A,B) fired only one or a few spikes at the onset of the stimulus and remained inactive thereafter. Phasic responses could clcarly be subdivided into two types, constant latency (Fig. $8 A$ ) and variable latency (Fig. 8B). Constant-latency neurons seldom fired more than one spike per stimulus, and this spike was tightly locked to the stimulus onset. Phasic variable-latency neurons fired one to three spikes at the 

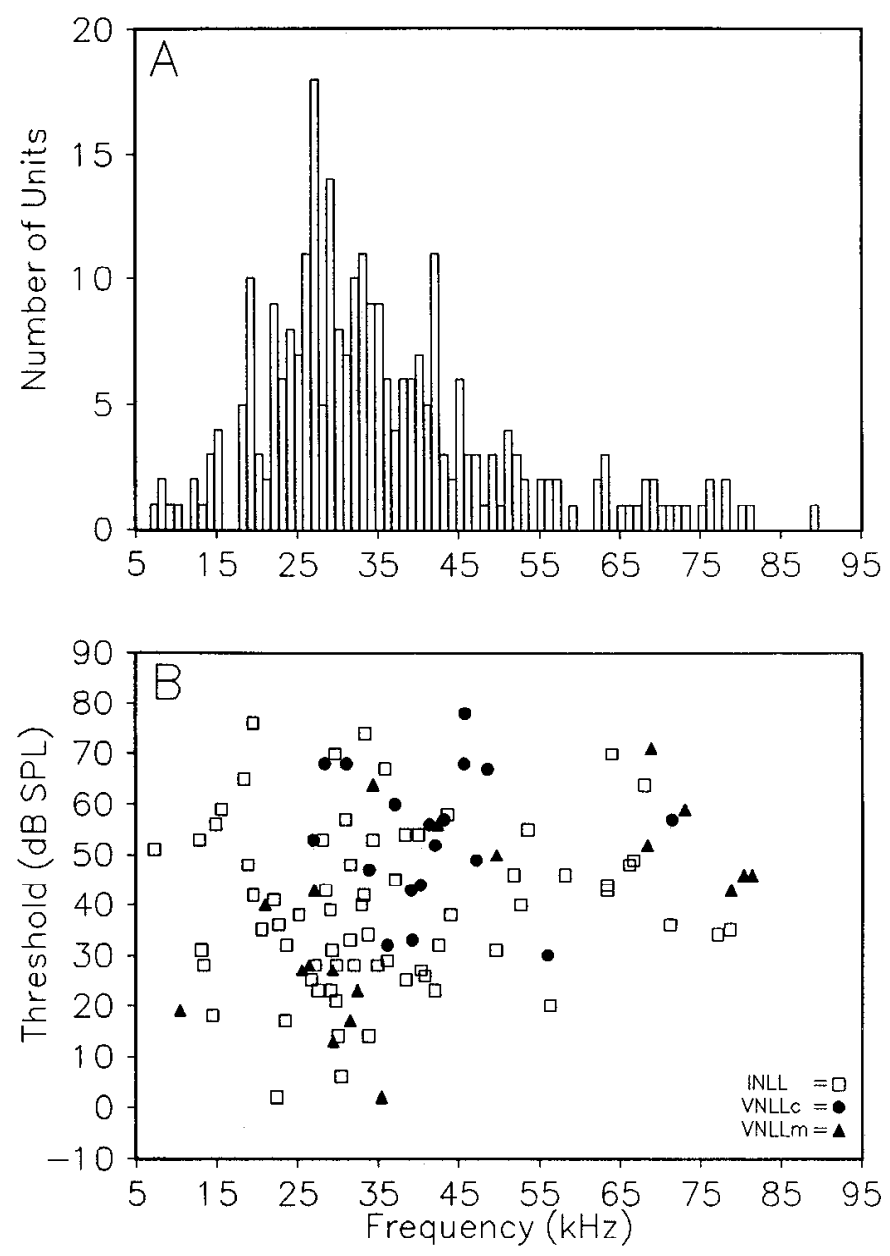

Figure 5. A, BFs for 88 single units in the three monaural nuclei of the lateral lemniscus are distributed throughout the entire range of frequencies audible to Eptesicus, but there is an expanded representation of the frequency range from 20 to $40 \mathrm{kHz}$, centered around $28 \mathrm{kHz}$. Because the frequency distributions in INLL, VNLLc, and VNLLm were very similar, data from all three nuclei are pooled to make the histogram. $B$. Pooled results from single units in INLL $(n=58)$, VNLLc $(n=15)$, and VNLLm $(n=15)$ to illustrate the relationship between minimum threshold and $\mathrm{BF}$. In each nucleus, the frequency range with the lowest minimum thresholds corresponded to the overrepresented range from 20 to $40 \mathrm{kHz}$.

onset of a stimulus, but the latency of the first spike varied by as much as several milliseconds from one stimulus presentation to another. Phasic neurons were found throughout the monaural nuclei of the lateral lemniscus, but there was a clear segregation of the constant-latency and variable-latency types. In the columnar nucleus, phasic constant-latency units were virtually the only type found, making up $95 \%$ of the population (Fig. 9). Phasic variable-latency neurons were found in INLL and VNLLm, where they made up about $20-30 \%$ of the total population.

"Choppers" (Fig. 8C) responded throughout the duration of the stimulus with spikes occurring at a regular ISI and tight locking of the first spike to the time of stimulus onset. The units that we classified as choppers mostly had ISIs of less than 2.5 msec and therefore were "fast" choppers (e.g., Tsuchitani, 1988a,b). They were found in INLL and VNLLm but not in VNLLc.

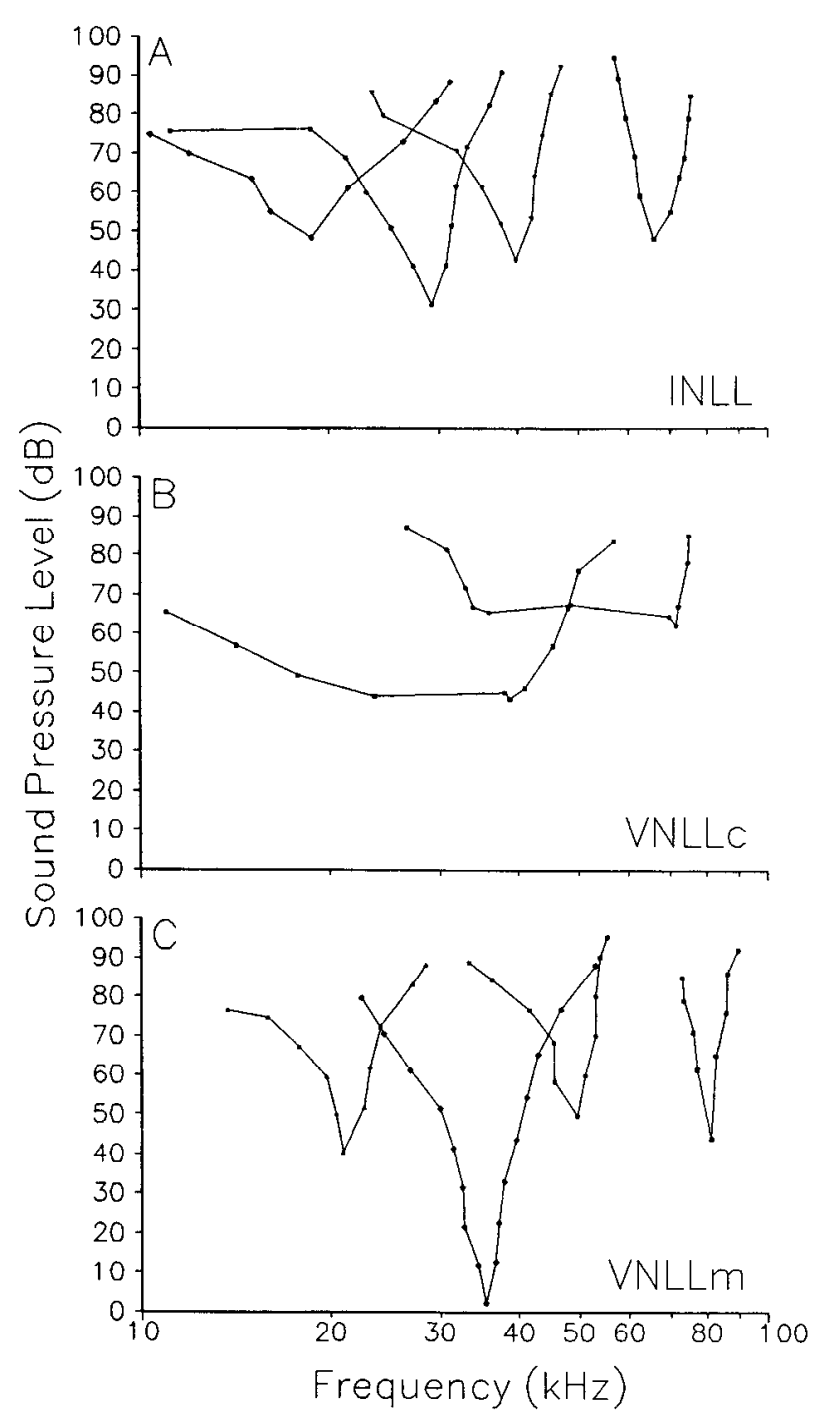

Figure 6. Representative tuning curves from the three monaural nuclei of the lateral lemniscus. Tuning curves in INLL $(A)$ and VNLLm $(C)$ are V-shaped and approximately symmetrical, whereas most tuning curves in VNLLc $(B)$ are broad and asymmetrical with a steep highfrequency slope and shallower low-frequency slope.

"Tonic" neurons (Fig. 8D) discharged at a constant high rate throughout the duration of the stimulus, with practically no adaptation even when the stimulus duration was long, that is, $50 \mathrm{msec}$ or more. They differed from choppers in that the first spike was poorly locked to the time of stimulus onset. Although the ISI for the majority of tonic units was irregular, some, such as the one shown in Figure $8 D$, fired with an ISI as regular as that of a chopper even though the first spike was not synchronized to the onset of the sound. For a few of these tonic neurons, the first spike became synchronized to onset at high sound levels, and they responded in a chopper pattern. Tonic neurons were only found in INLL and VNLLm.

A few units, which we classified as "primary-like" (Fig. 8E), responded throughout the stimulus duration, but firing rate diminished after an initial transient "on" response. Less than 5\% of units in each of the three monaural divisions of the lateral lemniscus responded in a primary-like pattern. 
"Pausers" (Fig. $8 F$ ) responded with an initial spike followed by an inactive period and then resumed sporadic firing at a diminished rate for the remainder of the stimulus duration. Pausers were only found in INLL and VNLLm, where they made up less than $4 \%$ of the population.

Thus, in INLL and VNLLm, response types are mixed. However, interposed between these two structures is the columnar nucleus, a cytoarchitecturally and physiologically unique region where virtually all responses are of a single type, phasic constant latency.

\section{Variability of first spike: neurons specialized for encoding onset}

The examples shown in Figure 8 suggest that for many neurons in the monaural nuclei of the lateral lemniscus, but particularly for VNLLc neurons (Fig. 8A), the variability in latency of the first spike was extremely low. To quantify this, we calculated the SD in latency of the first spike after stimulus onset, measured over 100 stimulus presentations under identical conditions (tone at $\mathrm{BF}, 5 \mathrm{msec}$ duration, $20 \mathrm{~dB}$ above threshold). Data were collected over a $20 \mathrm{msec}$ period, using a bin width of $1 \mu \mathrm{sec}$. The results show that the variability in latency was closely related to the neuron's discharge type and its location. The singlespike response of phasic neurons in VNLLc consistently provided a precise marker of stimulus onset. SDs for 13 VNLLc units ranged from 0.03 to $0.78 \mathrm{msec}$ (average, $0.14 \pm 0.2$ ). Choppers also provided good timing markers for onset; SDs in the time of occurrence of the first spike for 20 choppers ranged from 0.09 to $3.96 \mathrm{msec}$ (average, $0.77 \pm 0.84$ ). Although the sample of pauser neurons was small, only four neurons, they also appeared to have little variability in their first spike, with an average SD of $0.96 \mathrm{msec}$. In contrast, tonic, primary-like, and phasic variable-latency neurons were all poor markers of the time of stimulus onset. For all of these response classes, the SDs for first-spike latency averaged several milliseconds.

\section{Constant-latency neurons}

The phasic neurons in the VNI I c have an additional characteristic that makes them ideally suited to encode the onset of sound precisely - a response latency that is largely independent of changes in stimulus parameters. In order for a neuron to mark unambiguously the timing of an auditory event such as the onset of a sound, its latency should not only be stable under constant conditions, but ideally it should be independent of sound level and frequency. However, for most neurons in the auditory system, there are large and systematic changes in latency associated with changes in either sound level or frequency. To evaluate stability of latency under variable stimulus conditions, we measured latencies for 13 neurons in VNLLc, 34 in INLL, and 11 in VNLLm as stimulus parameters were varied. We defined constant-latency neurons as those having SDs in first-spike latency of less than $1.0 \mathrm{msec}$, and a change of less than $1.0 \mathrm{msec}$ in first-spike latency as SPL was increased from $10 \mathrm{~dB}$ above threshold to $40 \mathrm{~dB}$ above threshold. By these criteria, all VNLLc neurons tested were constant-latency responders. Three neurons in VNLLm (27\%) and 15 in INLL (44\%) were constant-latency responders, mainly pausers and choppers.

Figure 10 illustrates several differences between phasic VNLLc units and units that are not constant-latency responders. Two units in VNLLm are shown for comparison. First, as noted above, under each condition the SDs for the VNLLc unit (Fig. $10 A, B$ ) are far smaller than they are for the VNLLm units (Fig.

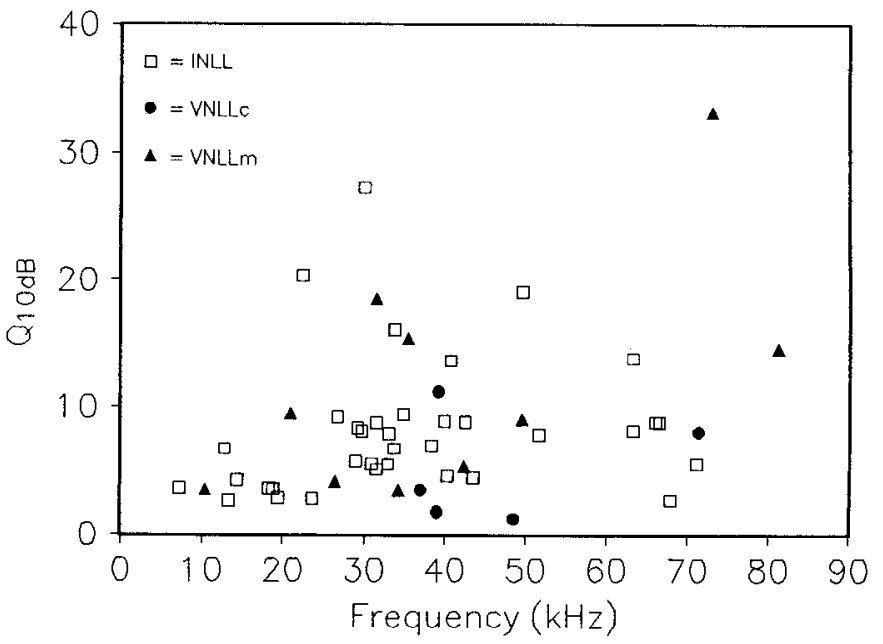

Figure 7. Plot of $Q_{10 \mathrm{~dB}}$ versus BF for single units in INLL $(n=36)$, VNLLc $(n=5)$, and VNLLm $(n=10)$ shows that breadth of tuning is not related to BF. However, neurons in VNLLc (solid circles) have, on the average, lower $Q_{10 \mathrm{~dB}}$ values than units in INLL or VNLLm.

$10 C, D)$. Second, when frequency was varied, the latency of the VNLLm units (Fig. 10C) increased by more than $1 \mathrm{msec}$ with a $10 \mathrm{kHz}$ shift away from BF; the latency of the VNLLc unit (Fig. 10A) remained virtually constant over a range of at least $30 \mathrm{kHz}$. When sound level was varied, the latency of the VNLLm unit (Fig. 10D) changed by approximately $2 \mathrm{msec}$ over a range of $30 \mathrm{~dB}$. Over the same range, the latency of the VNLLc unit (Fig. 10B) remained virtually constant. Thus, VNLLc neurons provide a very precise timing marker for the onset of a sound, and this marker is almost independent of the sound's spectral content and loudness. For this reason, we refer to the VNLLc units as phasic constant-latency responders.

Chopping rate and latency. Choppers (Fig. $8 \mathrm{C}$ ) fired very regularly near the beginning of the stimulus, but for many choppers the ISI appeared to become progressively more irregular throughout the duration of the sound. Increasing sound level typically resulted in greater regularity of chopping, but the rate of chopping was unaffected. Chopping rates, as expressed by ISI, ranged from 0.4 to $3.5 \mathrm{msec}$, with most falling in the range below $2 \mathrm{msec}$. Figure $11 \mathrm{~A}$ shows that there was a clear correlation between a neuron's latency and its chopping rate. Neurons with short latencies fired with a shorter ISI than did neurons with long latencies (Fig. 11A). This relationship suggests that chopping rate is determined by some biophysical property that also influences the latency of the initial spike. There was no correlation between the $\mathrm{BF}$ of a neuron and its rate of chopping (Fig. $11 B$ ), nor was chopping ratc affected by SPL.

Although most tonic neurons fired with an irregular ISI, some neurons that we classified as tonic fired with regular ISIs comparable to those of choppers. We did not classify them as choppers because the latency of the first spike was variable relative to the stimulus onset. Once the neuron began to fire, the interval between spikes remained stable. However, because of the variability in first-spike latency, the ISI appeared variable when data from multiple trials were combined to produce a PSTH. The response illustrated in Figure $8 D$ provides a good example of a unit that fired with a regular ISI, but with variable firstspike latency. 

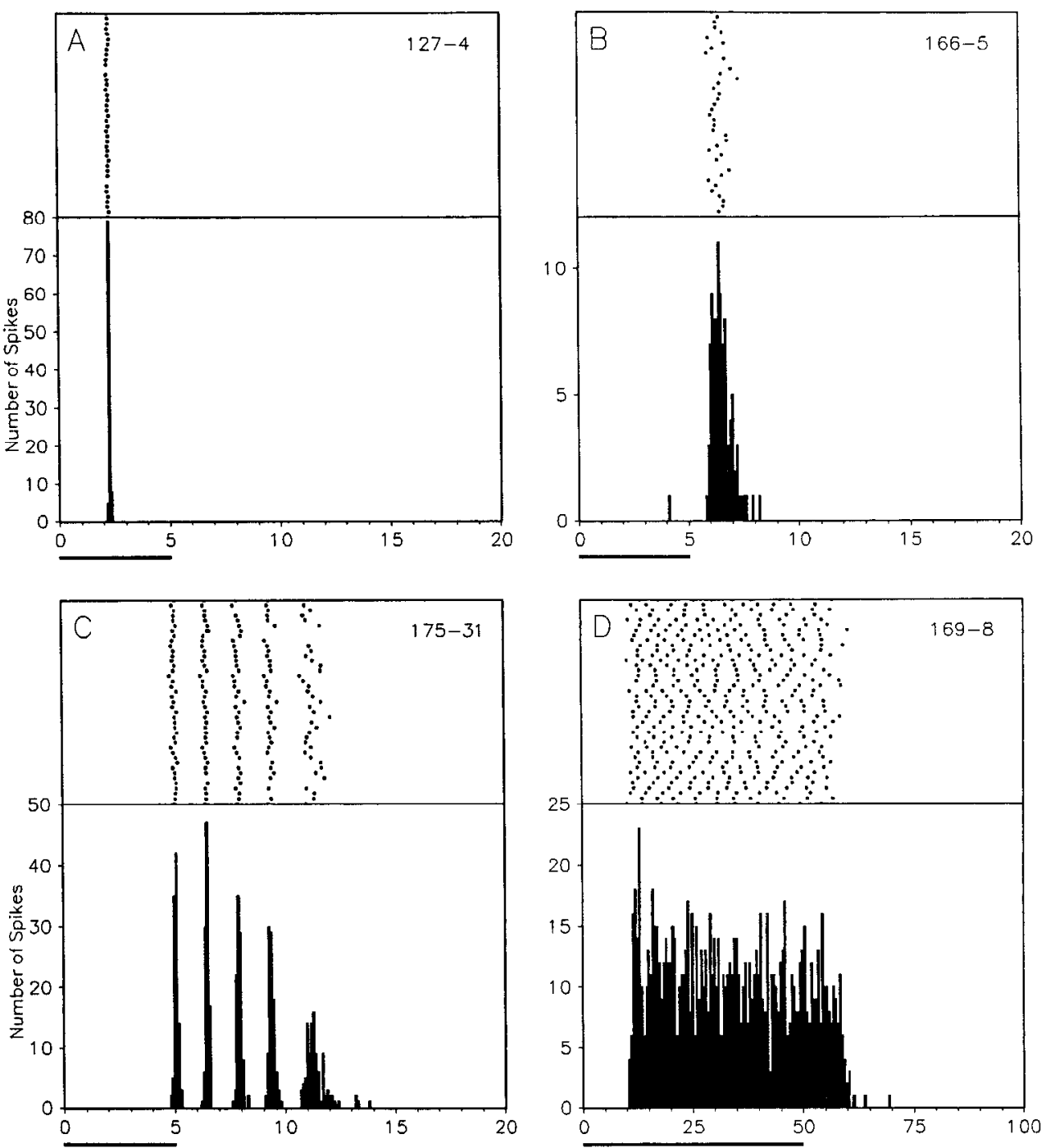

Figure 8. Typical discharge patterns of units in the monaural nuclei of the lateral lemniscus. Above each PSTH is a dot raster showing 40 of the 100 responses that make up the PSTH in the panel below. The bar below each PSTH shows stimulus duration. Unit numbers are given in the upper right corner of each panel. $A$, Phasic constant-latency neuron in VNLLc; $B$, phasic variable-latency neuron in INLL; $C$, chopper in VNLLm; $D$, tonic, nonadapting response in INLL; $E$, primary-like response in INLL; $F$, pauser response in VNLLm.
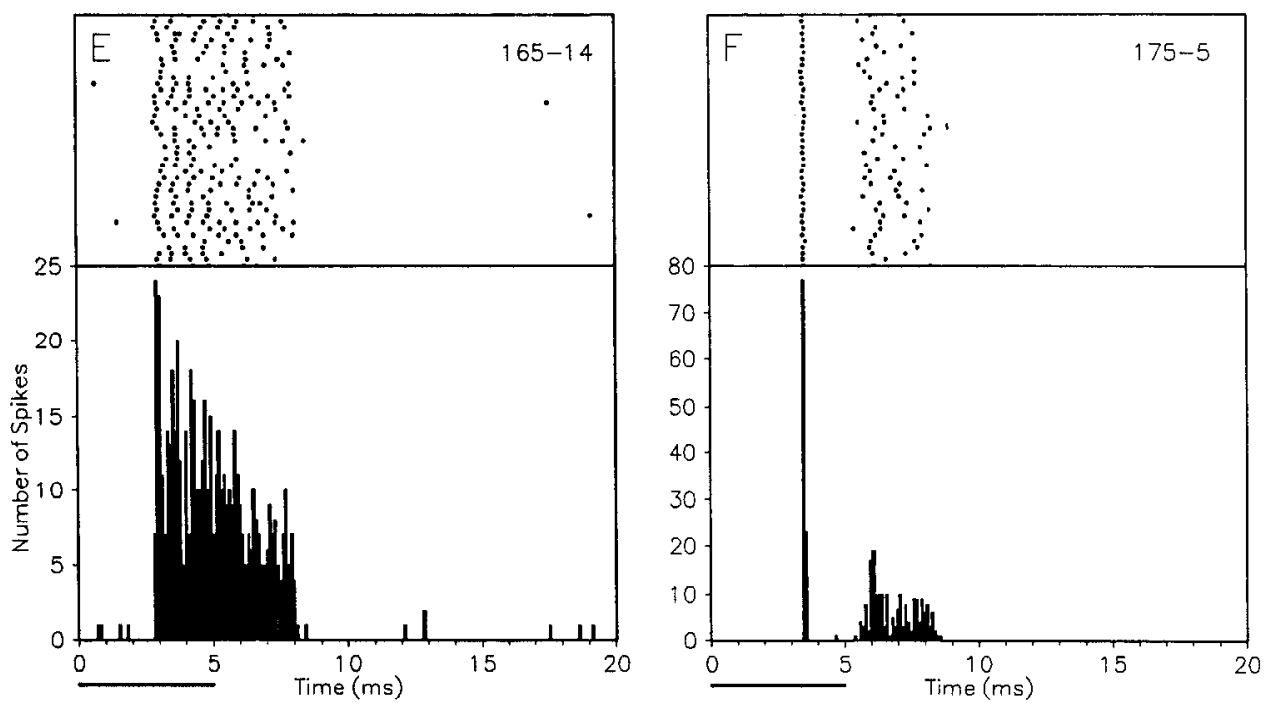

Latencies: INLL, VNLLc, and VNLLm have different latency ranges

Figure 12 shows that the latencies of VNLLc neurons were shorter than those in the other monaural lateral lemniscal nuclei.
In VNLLc, latencies ranged from 1.7 to $5.6 \mathrm{msec}$ (average, 3.3 $\pm 1.2 \mathrm{msec}$ ). Neurons in VNLLm had somewhat longer latencies, with a range of 3.3-6.3 msec (average, $4.4 \pm 1.1 \mathrm{msec}$ ). INLL neurons had both the widest range of latencies $(2.7-11.1$ $\mathrm{msec})$ and the longest average latency $(5.1 \pm 1.9 \mathrm{msec})$. Except 


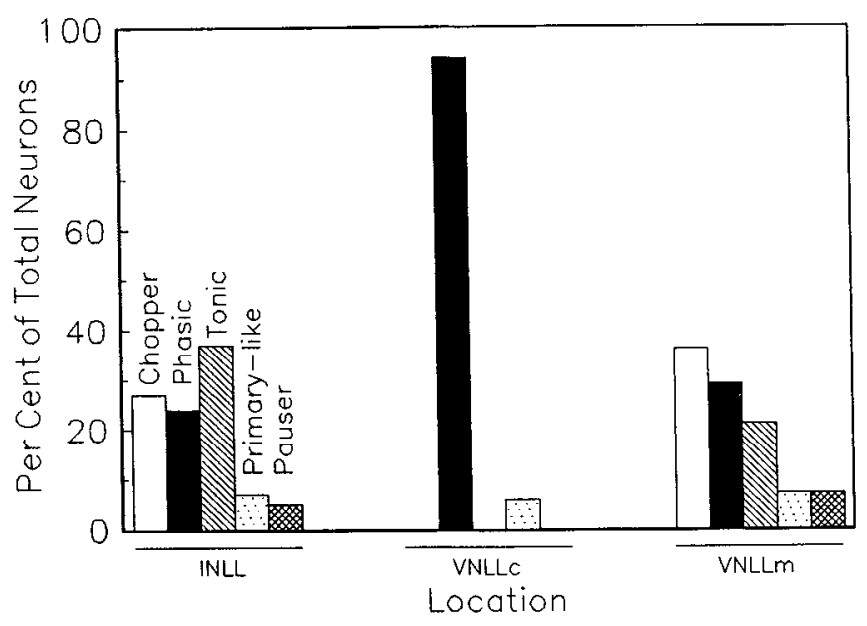

Figure 9. Distributions of discharge types in INLL $(n=59)$, VNLLc $(n=18)$, and VNLLm $(n=14)$. Responses in INLL and VNLLm are approximately evenly divided between chopper, tonic, and phasic variable-latency responses. Virtually all units in VNLLc are phasic constantlatency responders.

for the phasic constant-latency neurons in VNLLc and choppers in INL.I, and VNLLm, all of which had short latencies, there was no relation between latency and response type; thus, latency depended more on the location of the units than on their response type. The fact that the latency ranges for the different nuclei only partially overlap suggests the possibility for delay lines. That is, neurons in INLL, VNLLc, and VNLLm could provide a set of parallel pathways to the midbrain, the inputs of which are slightly offset in time at their target.

\section{Integration times: neurons in INLL, VNLLc, and VNLLm respond to short-duration sounds}

Virtually all of the neurons that we sampled in the monaural nuclei of the lateral lemniscus responded well to a $5 \mathrm{msec}$ stimulus; increasing stimulus duration had no effect on reliability of the response, or on threshold. Thus, $5 \mathrm{msec}$ was the standard duration that we used when presenting stimuli. In our entire sample, only one unit was encountered, in INLL, that required a stimulus longer than $5 \mathrm{msec}$ to elicit a reliable response. Because neurons in the nuclei of the lateral lemniscus appeared capable of responding to a very short stimulus, we tested a subset of 17 neurons with pure tone stimuli of variable duration to determine their minimum integration times, defined as the minimum stimulus duration necessary to elicit a reliable responsc comparable to that seen with a $5 \mathrm{msec}$ stimulus. Of these, 11 were in INLL, 3 were in VNLLc, and 3 were in VNLLm. Of the neurons tested with short-duration stimuli, all but one, in INLL, responded vigorously to a stimulus $1 \mathrm{msec}$ or less in duration. Thresholds for short stimuli were approximately the same as for the $5 \mathrm{msec}$ stimuli. For these neurons, minimum durations ranged from 0.05 to $1 \mathrm{msec}$, and the average was 0.3 $\pm 0.25 \mathrm{msec}$. This short integration time is very different from the intcgration times that we have measured in the central nucleus of the inferior colliculus (ICc) of Eptesicus using the same stimulus delivery system. In the ICc, many neurons require a pure tone stimulus $50 \mathrm{msec}$ or longer to evoke any response (Casseday and Covey, unpublished observations). This ability of neurons throughout the monaural nuclei of the lateral lemniscus to respond to a very short pure tone stimulus suggests that they are ideally suited to encode transient auditory events such as an FM sound sweeping rapidly through their range of frequency sensitivity, or short-duration amplitude peaks such

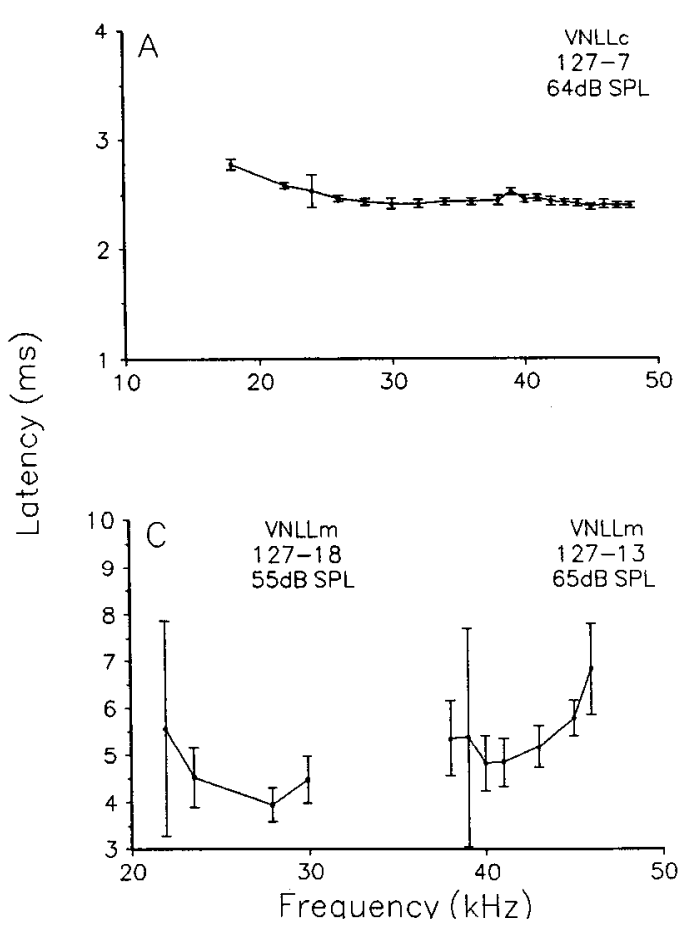

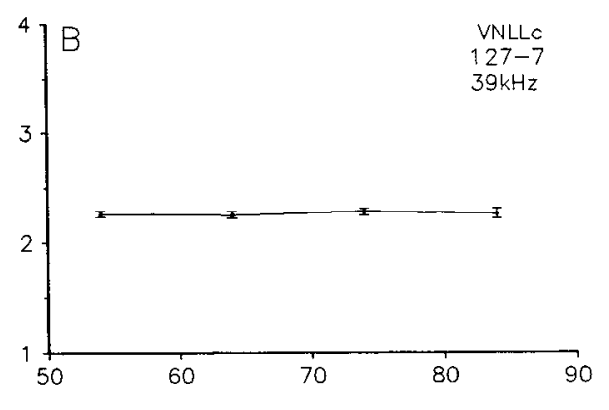

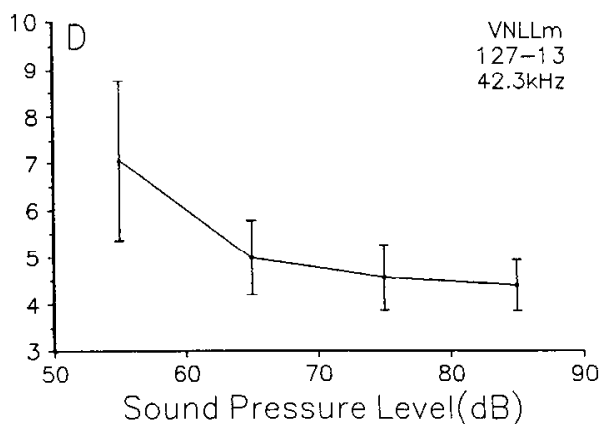

Figure 10. Responses of a constantlatency neuron in VNLLc $(A$ and $B$ ) compared with those of two neurons in VNLLm $(C$ and $D)$. Unit numbers are given above each graph. In the graphs on the left, latency is plotted as a function of frequency, measured at $20 \mathrm{~dB}$ above BF threshold. The neuron in VNLLc has a dynamic range of over 30 $\mathrm{kHz}$. Between 30 and $50 \mathrm{kHz}$, there is virtually no change in latency; latency increases slightly between 18 and $30 \mathrm{kHz}$ such that the latency at $18 \mathrm{kHz}$ is 0.39 msec longer than that at $50 \mathrm{kHz}$. In contrast, latency of VNLLm neurons changes by more than $1.5 \mathrm{msec}$ over a range of $10 \mathrm{kHz}$. In the graphs on the right, latency is plotted as a function of sound level. The VNLLc neuron responds with a constant latency over a range of more than $30 \mathrm{~dB}$. The latency of the VNI I $m$ neuron changes by more than $2.5 \mathrm{msec}$ over the same range. The error bars on the data points represent the SD over 100 stimulus presentations. The SDs of the VNLLc cell latencies are very small, between 0.02 and 0.04 msec. In contrast, the SDs for the VNLLm cell latencies are large, ranging up to several milliseconds. In order to show the error bars in $A$ and $B$, the $y$-axis scale is expanded relative to that in $C$ and $D$. 

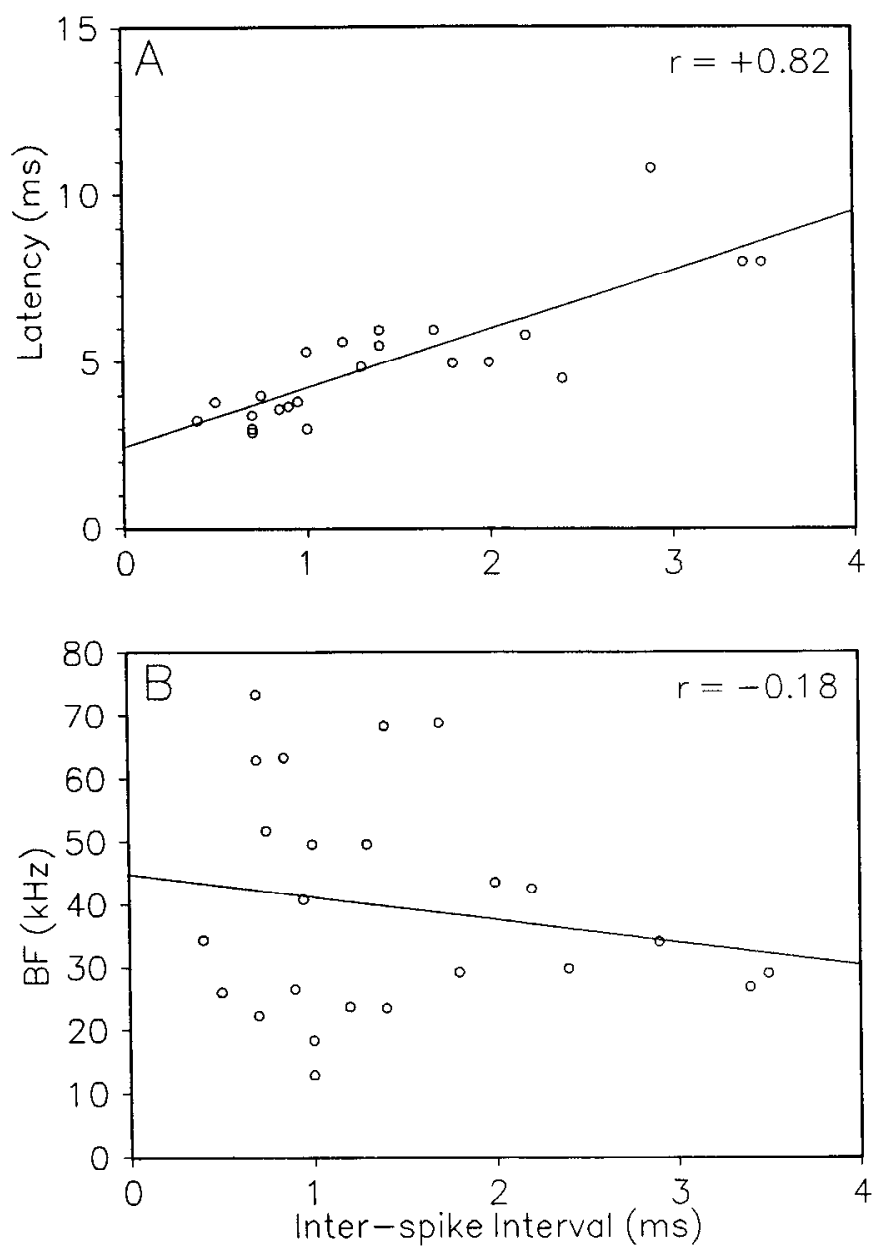

Figure 11. A, Plot of mean ISI and latency for choppers shows that there is a close relationship between the unit's latency and the interval between successive spikes throughout the response. $B$, Plot of latency versus $B F$ shows that latency is not related to $B F$ but is instead related to the location of the neuron. The shortest latencies are in VNLLc, and the longest, in INLL.

as are gencrated by reflection of echoes from three-dimensional objects.

This idea is further supported by our observation that all neurons in INLL, VNLLc, and VNLLm respond reliably to stimuli presented at high repetition rates. To obtain an initial idea of how rapidly neurons can follow amplitude modulations, we presented pairs of $5 \mathrm{msec}$ pure tone stimuli and determined recovery times following the first tone for a small sample of 10 neurons ( 6 in INLL, 3 in VNLLc, 1 in VNLLm). Minimum separation between the end of the first stimulus and the beginning of the second ranged from 0.1 to $3.7 \mathrm{msec}$ (mean, $1.4 \mathrm{msec}$ ). Although the above data on integration time and recovery time are limitcd, they are sufficient to indicate the importance of future experiments using stimuli with rapid frequency and amplitude modulations.

\section{Rate-level functions: most nonmonotonic neurons are choppers}

We measured discharge rate versus sound level functions for 58 single units, 34 of which were located in INLL, 13 in VNLLc, and 11 in VNLLm. This was done by presenting a 5 msec pure tone stimulus at the unit's BF, starting at $10 \mathrm{~dB}$ above threshold and increasing sound level in $10 \mathrm{~dB}$ steps. We collected data

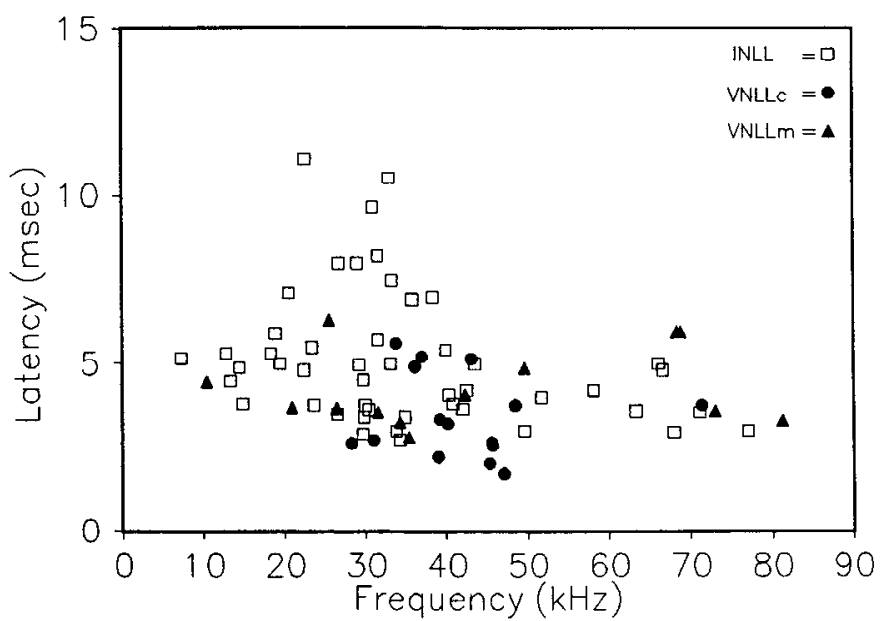

Figure 12. Distribution of latencies in response to pure tones at BF, $10 \mathrm{~dB}$ above threshold, for neurons in INLL $(n=50)$, VNLLc $(n=15)$, and VNLLm $(n=14)$. Note that the shortest latencies are in VNLLC (solid circles).

from 100 stimulus presentations at each sound level. To facilitate comparison among units with very different firing rates, the data for each neuron were normalized to its own maximum evoked spike rate, which was designated $100 \%$ of maximum rate. We defined monotonic functions as those in which an increase in sound level resulted in an increased or stable firing rate; we defined nonmonotonic functions as those in which an increase in sound level at some point resulted in a decrease in firing rate of at least $10 \%$. By these criteria, $59 \%$ of all units in our sample had monotonic rate-level functions, and $41 \%$ had nonmonotonic rate-level functions. The results indicated that the differences were more closely related to response type than to location (Fig. 13).

All phasic units, whether constant or variable latency, had monotonic rate-level functions; the difference between the two classes was in the steepness of the slope. Nearly all of the constant-latency responders in VNLLc (Fig. 13A) had rate-level relationships that were nearly step functions, virtually flat above threshold. Such units had a dynamic range of $10 \mathrm{~dB}$ or less over which firing rate increased, responding at or near their maximum of one spike per stimulus at all suprathreshold sound levels. Such step functions were seldom seen for other discharge types. This type of rate-intensity function would be particularly poor for encoding sound level over wide ranges but would ensure that a reliable timing signal is transmitted at all sound levels above the neuron's threshold. Phasic variable-latency neurons had a broader dynamic range, approximately 20-30 dB, over which firing rate increased.

The majority (79\%) of tonic units in both INLL and VNLLm had monotonic rate-level functions. Although a few tonic neurons had steep rate-level functions (see Fig. 13C), most had relatively broad dynamic ranges, between 20 and $60 \mathrm{~dB}$, over which firing rate increased. The fact that tonic neurons have the most gradual slopes and widest dynamic range of response to varying sound level suggests that they are the best suited to encode sound level information. The remaining $21 \%$ of tonic neurons had nonmonotonic rate-level functions.

Over half (54\%) of all choppers (Fig. 13B) had nonmonotonic rate-level functions. The choppers were located either in INLL or in VNLLm. Neurons with these nonmonotonic responses would be somewhat ambiguous encoders of sound level, al- 
though they might respond as well or better to echoes at low sound levels than to echolocation pulses emitted at high sound levels.

\section{Discussion}

The present results have shown that neurons in INLL, VNLLc, and VNLLm are monaural; almost without exception they are excited by sound at the contralateral ear and unaffected by sound at the ipsilateral ear. In addition, they all share certain characteristics that make them especially well suited to encode temporal information. They have little or no spontaneous activity, they are broadly tuned, and they have short integration times. All of these findings, taken together with previous connectional evidence, indicate that the INLL, VNLLc, and VNLLm can be considered collectively as a unitary system of monaural pathways to the midbrain and suggest that a common function of these pathways is analyzing and encoding information about the temporal structure of sounds. In short, we propose that INLL, VNLLc, and VNLLm are components of an integrated system for processing of temporal information, just as the medial and lateral superior olives are part of an integrated system for binaural analysis.

The present results also provide evidence that within the broad functional context of temporal analysis, there is a division of labor such that each nucleus performs a different specialized analytical task and gives rise to a separate ascending pathway. The first evidence for this is the finding that INLL, VNLLc, and VNLLm each has its own complete tonotopic representation. Second, there are differences in response properties among the three monaural cell groups, suggesting that neurons in VNLLc encode information about the onset time of a sound while those in INLL and VNLLm encode information about temporal features of an ongoing sound.

We shall first consider the specialized functions of the individual pathways and then the function of the system as a whole.

\section{Specialized pathways for temporal analysis}

Onset processing in VNLLc. The phasic constant-latency neurons in VNLLc show the clearest and most obvious evidence of specialization to encode temporal information. Here, we discuss the possible mechanisms that contribute to the constantlatency response and the possible role of constant-latency units in auditory processing.

Most neurons in VNLLc fire one spike at the onset of every suprathreshold stimulus. The latency of this response is invariant even when the frequency or level of a sound is varied over a wide range. Thus, the response is an all-or-nothing signal of stimulus onset, precisely locked to this specific event. However simple this response is, it is not a simple matter for the nervous system to achieve. For most auditory neurons, latency decreases as sound level increases. Latency is also normally shortest at BF and longer as frequency deviates from BF.

The results suggest a partial answer as to how the constantlatency response may be produced. The broad, flat shape of tuning curves in VNLLc suggests two factors that may contribute to latency stability: convergence across frequency and a relatively high excitatory threshold for the VNLLc cell. Anatomical evidence supports the idea of convergent input across a wide range of frequencies. In an earlier study (Covcy and Casseday, 1986), we showed that the AVCN projects in a very systematic way to sheets of cells in VNLI.c; there is a precise correspondence between the anatomical locations of projection sheets in
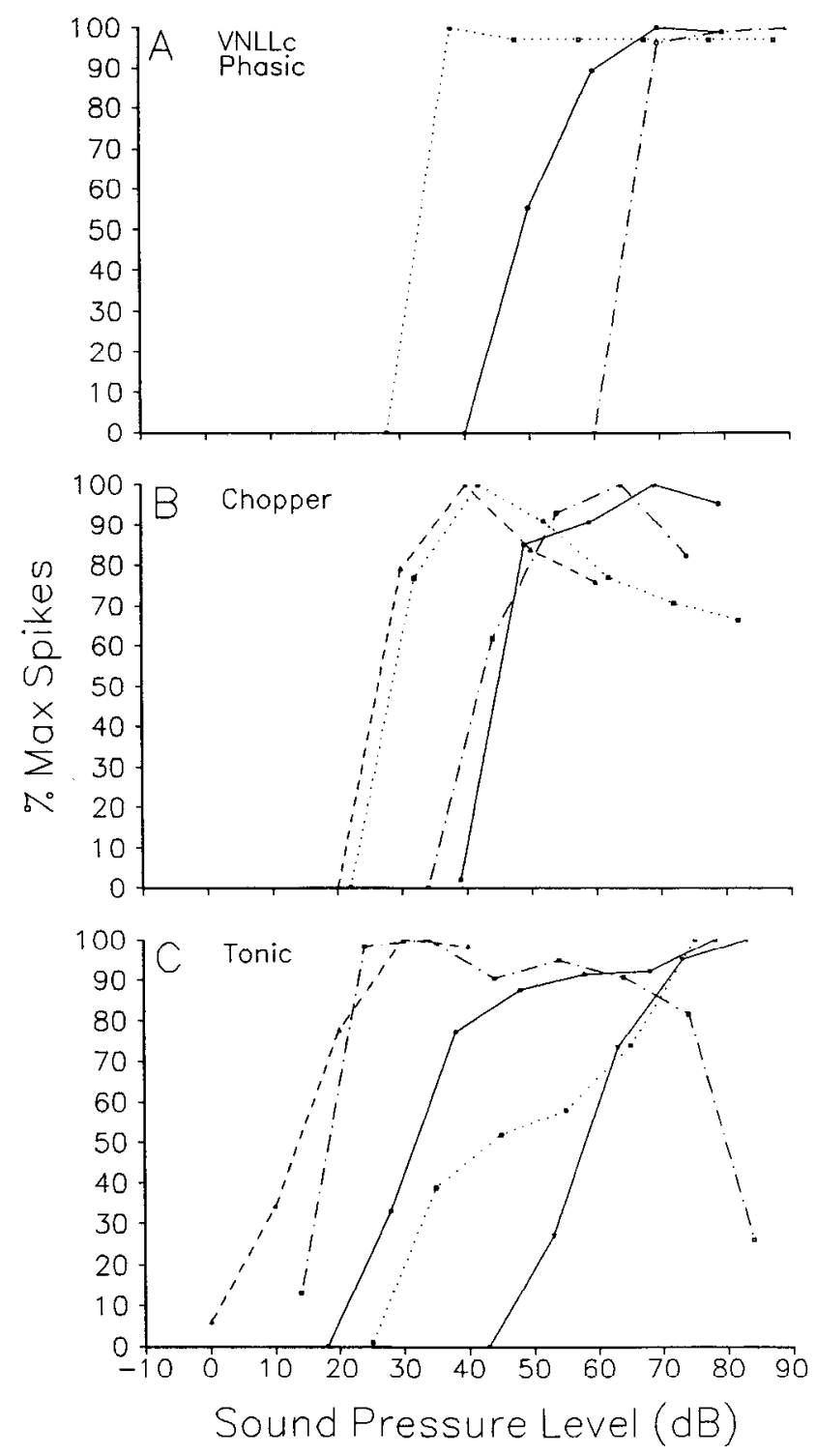

Figure 13. Typical rate-level functions for classes of neurons with different discharge patterns. $A$, For most phasic constant-latency units in VNLLc, the maximum firing rate of 1 spike per stimulus is reached as soon as sound level is a few decibels above threshold. $B$, Many choppers have nonmonotonic rate-level functions. They attain their maximum firing rate rapidly, within 10-20 dB above threshold, but further increases in sound level result in decreased firing. $C$, Tonic responders typically reach maximum firing rate morc slowly than do units in other response classes, with gradually increasing rate over a range of 30-40 dB above threshold. For some tonic neurons, rate-level functions are nonmonotonic, but the majority are monotonic.

VNLLc and the tonotopic locations of WGA-HRP injections in the AVCN. The precision of this correspondence was puzzling in view of the variable nature of WGA-HRP injections. The broad tuning curves of cells in VNLLc suggest an explanation. That is, there must be considerable convergence from the AVCN to sheets of cells in VNLLc. There are less than 20 projection sheets in VNLLc, each one only a single cell thick, stacked from dorsal to ventral. If the projections from AVCN were equally distributed and nonoverlapping, each sheet would represent about $4 \mathrm{kHz}$, that is, $1 / 20$ of the bat's hearing range, $10-90 \mathrm{kHz}$ (Dalland, 1965). However, the breadth of tuning in VNLLc 
neurons is far greater than a $4 \mathrm{kHz}$ band, even near threshold. A single VNLLc neuron may respond to a range as great as 40 $\mathrm{kHz}$ at just $10 \mathrm{~dB}$ above threshold; that is, it must receive input from a broad range of frequencies. Thus, at a few decibels above threshold, the frequencies represented in individual sheets must overlap considerably.

The broad, flat tuning of VNLLc neurons at near threshold levels suggests that there is some mechanism that makes a VNLLc neuron less selective for frequency than are the neurons that project to it. The broad tuning, together with the observation that VNLLc neurons tend to have relatively high excitatory thresholds, suggests that they respond only when the source or sources of their input are responding at levels well above threshold, in the broad part of their tuning curves, and thus at a level where latency shifts are minimal. There is at present no evidence to determine whether the flat sensitivity of VNLLc neurons is an intrinsic property, due to high synaptic thresholds, or whether it is due to external inhibitory processes. The projections from AVCN to VNLLc terminate as large calyccal endings on ccll bodies (Covey and Casseday, 1986); immunostaining with an antibody to glycine reveals that cell bodies in VNLLc are surrounded by large glycine-positive terminals (Covey and Casseday, unpublished observations). To understand fully the mechanisms responsible for the constant-latency response, it will be important to learn more about the patterns of input to VNLLc neurons.

A related unresolved issue is the mechanism by which one and only one spike occurs in response to a stimulus. If this is not simply the reflection of a single, phasic input, it seems likely that inhibitory processes are responsible, possibly originating in the medial nucleus of the trapezoid body, which is known to project to the intermediate and ventral nuclei of the lateral lemniscus (Zook and Casseday, 1982b; Spangler et al., 1985). Alternatively, membrane properties of the cell could render it unresponsive for a long period after discharging one action potential.

Constant-latency units similar to those we describe in VNLLc have also been seen in the ICc of echolocating bats (Suga, 1970; Pollak et al., 1977; Bodenhamer et al., 1979; Bodenhamer and Pollak, 1981). The present results indicate that the constantlatency response originates below the level of the ICc. Suga and Schlegel (1973) described a neuron in the lateral lemniscus of the gray bat, Myotis grisescens, which had very small SDs in latency, around $0.02 \mathrm{msec}$. Whether constant-latency properties originate in the VNLLc or at a prior level is still an open question. At the present time, we cannot entirely rule out the possibility that VNLLc neurons might simply reflect the responses of phasic neurons at a more peripheral level. However, there have been no reports of constant-latency responses at the cochlear nucleus or auditory nerve.

The finding that VNLLc is composed almost exclusively of constant-latency responders has significance for echolocation. VNLLc neurons appear to function as a set of partially overlapping broad-band filters, each of which gives an all-or-none signal that is perfectly correlated with the moment when a stimulus enters its range of sensitivity. Eptesicus uses an echolocation call that sweeps from high to low frequencies. The duration and frequency parameters of the call vary depending on the bat's behavior; when the bat is actively pursuing an insect, the most intense, first harmonic sweeps from about $60 \mathrm{kHz}$ to $25 \mathrm{kHz}$ in 3-5 msec (Simmons, 1989). We can predict the neural response to echolocation sounds based on both the data of Bodenhamer and Pollak (1981) on constant-latency neurons in the ICc, and our own testing of constant-latency neurons in VNLLc using FM sweeps. Cells in any given projection sheet in VNLLc should fire at the moment the sweep enters the upper range of their tuning curves. Thus, there would be a successive ventral-todorsal activation of the sheets during the vocalized call; there would be a second wave of activation, in the same sequence, during the returning echo. The time delay between the response to the pulse and the response to the echo is proportional to the target distance. Thus, VNLLc could provide accurate markers for pulse-echo delays in a number of different channels. In this way, VNLLc may represent the timing input for the neural comparators for measuring target range.

Choppers and tonic responders. Although the potential functions of tonic and chopping neurons are not as obvious as those of phasic constant-latency neurons in VNLLc, all chopper and most tonic neurons in INLL and VNLLm possess characteristics that provide good temporal resolution: low spontaneous activity, broad frequency tuning, and short integration time. In addition, choppers are precise markers of the onset of a sound. The fact that different neurons have different chopping rates raises the possibility that there are different channels, each with its own "clock rate." It remains to be seen whether the rate of chopping is synchronized with some aspect of the stimulus. One possibility is amplitude and frequency modulations that are present in echoes from textured objects.

Integration of the monaural pathways in the inferior colliculus. It should be clear from the temporal response properties of INLL, VNLLc, and VNLLm that they represent only an initial stage of processing and that their outputs must be integrated at a higher level. The INLL, VNLLc, and VNLLm, like the medial superior olive (MSO) and the lateral superior olive (LSO), project to the central nucleus of the inferior colliculus (see Pollak and Casscday, 1989, for review). In fact, in the big brown bat, we know of no other target of these nuclei. Moreover, there is evidence that frequency-specific projections from each of the monaural nuclei all terminate in the same region in the central nucleus of the inferior colliculus (Zook and Casseday, 1982b, 1987; Covey and Casseday, 1986; Ross et al., 1988). This means that there could be convergence of inputs from neurons with onsel, chopper, and tonic response types, all within a very restricted part of the inferior colliculus, perhaps on a single cell.

This observation raises the possibility that the various parts of the ventral lateral lemniscus constitute elements in a complex system of delay lines to the inferior colliculus. If the different monaural nuclei of the lateral lemniscus provide convergent inputs to a single target in the ICc, neurons whose latency falls into different ranges could provide a series of signals that arrive at different times at their targets on midbrain neurons. The target ncurons could encode specific time delays or temporal patterns of sound. That this is a reasonable idea is suggested by findings of ICc neurons whose response to the second of a pair of sounds is either inhibited or facilitated (Suga and Schlegel, 1973; Grinnell and Brown, 1978) and neurons that are selective for specific rates of amplitude modulation (Langner and Schreiner, 1988; Schreiner and Langner, 1988).

Comparison with the binaural system. The monaural nuclei of the lateral lemniscus are comparable to the superior olivary nuclei both in their size and in the magnitude of their projection to the inferior colliculus (Adams, 1979a). Like the nuclei of the 
superior olive, the monaural nuclei of the lateral lemniscus represent a stage of processing interposed between the cochlear nucleus and the inferior colliculus. Thus, the pathways that originate in INLL, VNLLc, and VNLLm are equivalent in position and parallel to the pathways of the superior olivary nuclei.

There is a large body of evidence that shows that, in most mammals, the LSO and MSO give rise to functionally specialized binaural pathways to the inferior colliculus. Many anatomical and physiological experiments in nonecholocating mammals have demonstrated that LSO and MSO process the two major cues for sound localization, interaural sound level differences in LSO, and interaural time differences in MSO (e.g., Stotler, 1953; Galambos et al., 1959; Warr, 1966, 1982; Goldberg and Brown, 1969; Boudreau and Tsuchitani, 1970; Guinan et al., 1972a,b; Tsuchitani, 1977; Glendenning et al., 1985). Studies in the barn owl have also shown a separation of brainstem pathways for analysis of interaural sound level differences and interaural time differences (e.g., Takahashi et al., 1984). In the bat, the situation is somewhat different; although the LSO is a binaural structure, the MSO is mainly monaural and probably serves a specialized function unique to echolocation (Casseday et al., 1988; Covey et al., 1991). Nevertheless, even in the bat, the MSO and LSO are clearly separate parallel pathways with different specialized functions. Anatomical studies have shown that the LSO and MSO of all mammals give rise to separate parallel pathways, all of which terminate at the inferior colliculus (e.g., Roth et al., 1978; Schweizer, 1981; Glendenning and Masterton, 1983; Willard and Martin, 1983; Zook and Casseday, 1987; Casseday et al., 1988). Although there is specialization of function within each pathway, the superior olivary pathways of nearly all mammals subserve a broader common function, comparison of sound at the two ears.

Similarly, the monaural nuclei of the lateral lemniscus give rise to a set of parallel pathways to the inferior colliculus. The anatomical cvidence for scparatc pathways originating in these nuclei is quite clear. The INLL, VNLLc, and VNLLm all receive divergent projections from the cochlear nucleus; each is in turn the origin of a separate pathway that terminates in the inferior colliculus (Zook and Casseday, 1982b, 1985; Covey and Casseday, 1986; present study). We propose that the set of pathways via the monaural nuclei of the lateral lemniscus all share a common function in that they are concerned with the analysis and encoding of temporal information; at the same time, each is functionally specialized for some different aspect of this analysis. At present, it is not known whether the different monaural pathways converge with one another at the level of single cells in the inferior colliculus, nor is it known whether there is convergence of monaural and binaural pathways on single cells. However, the fact that all of these pathways terminate in the same general area suggests that there might be such convergence.

\section{Are the monaural nuclei of the lateral lemniscus unique to the bat?}

Structure and connections. All of the previously published evidence in both bats and other mammals suggests that the monaural lateral lemniscal nuclei comprise multiple structurally distinct divisions. It has been shown that the ventral lateral lemniscal region of the cat contains a variety of different cell types that are at least partly segregated (Adams, 1979a; Glendenning et al., 1981). In the bat, the different cell types are extremely well segregated but do not appear to be substantially different in their morphology from those in cat (Zook and Casseday, 1982a; Covey and Casseday, 1986; present study). For example, in the cat, Adams (1979a) describes multipolar cells, "horizontal cells" that resemble those in INLL of the bat, and "small oval" cells similar to those in the medial nucleus of the trapezoid body; these oval cells are very similar to those in VNLLc of the bat. This comparison suggests that VNLLc is not unique to the bat, but is present, at least in rudimentary form, in all mammals. In both cat and bat, anterograde transport from the cochlear nucleus takes the form of multiple patches in the lateral lemniscal nuclei, indicating that in both species there is divergence of input from the cochlear nucleus to multiple areas in the lateral lemniscal nuclei (Warr, 1966, 1982; Glendenning et al., 1981; Covey and Casseday, 1986). In all mammals studied, retrograde transport from the inferior colliculus results in multiple patchlike clusters of labeled cells in the lateral lemniscal nuclei, indicating multiple sources of projections to a single area within the inferior colliculus (Roth et al., 1978; Adams, 1979a; Brunso-Bechtold et al., 1981; Zook and Casseday, 1982b; Covey and Casseday, 1986).

Tonotopy. The divergence of afferents to patches within the lateral lemniscal nuclei in the cat and bat indicates the likelihood of multiple tonotopic representations. Studies on tonotopy in the cat are based on small samples of single units. In one study, a dorsal-to-ventral progression was seen (Aitkin et al., 1970), and in the other no tonotopic organization was seen (Guinan et al., 1972a,b). In view of the more recent anatomical findings, it is very possible that multiple tonotopic representations could have been missed because of the small sample sizes. In view of the multiple tonotopic representations seen now in the lateral lemniscus of two species of bats (Metzner and Radtke-Schuller, 1987; present study), it would be useful to reexamine tonotopy in the lateral lemniscus of nonecholocating mammals.

Response properties. The above two studies on the cat also provided enough information on response properties of neurons in the intermediate and ventral lateral lemniscal nuclei to suggest similarities with the present findings. Both studies indicate that most of the neurons located in the ventral lateral lemniscus were monaural, excited by the contralateral ear. Phasic and sustained response types were reported in both studies. Guinan et al. (1972b) reported that out of 23 units, 14 had a chopper pattern, 6 were phasic-on, and 3 were tonic. Moreover, 15 out of 22 neurons had V-shaped tuning curves, and 2 had "wide" tuning curves.

Evidence for a binaural area in the ventral lateral lemniscus of cats seems to be the major respect in which the lateral lemniscus of cats differs from that of bats. Guinan et al. (1972b) reported that some neurons were binaural, excited by the contralateral ear and inhibited by the ipsilateral ear. Connectional studies reveal a small binaural part of the ventral nucleus of the lateral lemniscus in the cat (Glendenning et al., 1981).

We conclude with the hypothesis that the intermediate and ventral nuclei of the lateral lemniscus are basically similar in all mammals. The only exception to this idea is a small binaural area in the ventral nucleus of the cat. We further propose that the function of the monaural pathways in all mammals is similar, but in the bat they are more highly developed to deal with the extraordinary demands made by echolocation. The echolocating bat is not the only species that must analyze complex temporal patterns of sound. The ability to discriminate among different temporal sequences of sounds is crucial to behaviors 
as diverse as hunting insects by echolocation and understanding human speech.

\section{References}

Adams JC (1979a) Ascending projections to the inferior colliculus. J Comp Neurol 183:519-538.

Adams JC (1979b) A fast, reliable silver-chromate Golgi method for perfusion-fixed tissue. Stain Tech 58:225-226.

Aitkin LM, Anderson DJ, Brugge JF (1970) Tonotopic organization and discharge characteristics of single neurons in the nuclei of the lateral lemniscus of the cat. J Neurophysiol 33:421-440.

Bodenhamer RD, Pollak GD (1981) Time and frequency domain processing in the inferior colliculus of echolocating bats. Hearing Res 5:317-335.

Bodenhamer RD, Pollak GD, Marsh DS (1979) Coding of fine frequency information by echoranging neurons in the inferior colliculus of the Mexican free-tailed bat. Brain Res 171:530-535.

Boudreau JC, Tsuchitani C (1970) Cat superior olive S-segment cell discharge to tonal stimulation. In: Contributions to sensory physiology, Vol 4 (Neff WD, ed), pp 143-213. New York: Academic.

Brugge JF, Anderson DJ, Aitkin LM (1970) Responses of neurons in the dorsal nucleus of the lateral lemniscus of cat to binaural tonal stimulation. J Neurophysiol 33:441-458.

Brunso-Bechtold JK, Thompson GC, Masterton RB (1981) HRP study of the organization of auditory afferents ascending to central nucleus of inferior colliculus in cat. J Comp Neurol 197:705-722.

Casseday JH, Covey E, Vater M (1988) Connections of the superior olivary complex in the rufous horseshoe bat, Rhinolophus rouxi. J Comp Neurol 278:313-329.

Covey E, Casseday JH (1986) Connectional basis for frequency representation in the nuclei of the lateral lemniscus of the bat Eptesicus fuscus. J Neurosci 6:2926-2940.

Covey E, Vater M, Casseday JH (1991) Binaural properties of single units in the superior olivary complex of the mustache bat. J Neurophysiol, in press.

Dalland JI (1965) Hearing sensitivity in bats. Science 150:1185-1186.

Galambos R, Schwartzkopff J, Rupert A (1959) Microelectrode study of superior olivary nuclei. Am J Physiol 197:527-536.

Glendenning KK, Masterton RB (1983) Acoustic chiasm: efferent projections of the lateral superior olive. J Neurosci 3:1521-1537.

Glendenning KK, Brunso-Bechtold JK, Thompson GC, Masterton RB (1981) Ascending afferents to the nuclei of the lateral lemniscus. J Comp Neurol 197:673-703.

Glendenning KK, Hutson KA, Nudo RJ, Masterton RB (1985) Acoustic chiasm. II. Anatomical basis of binaurality in lateral superior olive of cat. J Comp Neurol 232:261-285.

Goldberg JM, Brown PB (1969) Response of binaural neurons of dog superior olivary complex to dichotic tonal stimuli: some physiological mechanisms of sound localization. J Neurophysiol 32:613-636.

Grinnell AD, Brown P (1978) Long-latency "subthreshold" collicular responses to the constant-frequency components emitted by a bat. Science 202:996-999.

Guinan JJ Jr, Guinan SS, Norris BE (1972a) Single auditory units in the superior olivary complex. I. Responses to sounds and classifications based on physiological response properties. Int $J$ Neurosci 4: 101-120.

Guinan JJ Jr, Norris BE, Guinan SS (1972b) Single auditory units in the superior olivary complex. II. Locations of unit categories and tonotopic organization. Int J Neurosci 4:147-166.

Langner G, Schreiner CE (1988) Periodicity coding in the inferior colliculus of the cat. I. Neuronal mechanisms. J Ncurophysiol 60: $1799-1822$

Mesulam M-M (1978) Tetramethylbenzidine for horseradish peroxidase neurochemistry: a non-carcinogenic blue reaction product with superior sensitivity for visualizing afferents and efferents. J Histochem Cytochem 26:106-117.

Metzner W, Radtke-Schuller S (1987) The nuclei of the lateral lemniscus in the rufous horseshoe bat, Rhinolophus rouxi. J Comp Physiol A $160: 395-411$.

Pfeiffer RR (1966) Classification of response patterns of spike discharges for units in the cochlear nucleus: tone-burst stimulation. Exp Brain Res 1:220-235.
Poljak S (1926) Untersuchungen am Oktavussystem der Säugetiere und an den mit diesem koordinierten motorischen Apparaten des Hirnstammes. J Psychol Neurol 32:170-231.

Pollak GD, Casseday JH (1989) The neural basis of echolocation in bats. Berlin: Springer.

Pollak GD, Marsh DS, Bodenhamer R, Souther A (1977) Echo-detecting characteristics of neurons in inferior colliculus of unanesthetized bats. Science 196:675-678.

Ross LS, Pollak GD, Zook JM (1988) Origin of ascending projections to an isofrequency region of the mustache bat's inferior colliculus. $J$ Comp Neurol 270:488-505.

Roth GL, Aitkin LM, Andersen RA, Merzenich MM (1978) Some features of spatial organization of the central nucleus of the inferior colliculus of the cat. J Comp Neurol 182:661-680.

Schreiner CE, Langner G (1988) Periodicity coding in the inferior colliculus of the cat. II. Topographical organization. J Neurophysiol 60:1823-1840.

Schweizer H (1981) The connections of the inferior colliculus and the organization of the brainstem auditory system in the greater horseshoe bat Rhinolophus ferrumequinum. J Comp Neurol 201:25-49.

Simmons JA (1989) A view of the world through the bat's ear: the formation of acoustic images in echolocation. Cognition 33:155-199.

Spangler KM, Warr WB, Henkel CK (1985) The projections of principal cells of the medial nucleus of the trapezoid body in the cat. $J$ Comp Neurol 238:249-262.

Stensaas LJ, Stensaas SS (1968) Astrocytic neuroglial cells, oligodendrocytes and microgliacytes in the spinal cord of the toad. Z Zellforsch $84: 473-489$

Stotler WA (1953) An experimental study of the cells and connections of the superior olivary complex of the cat. J Comp Neurol 98:401432.

Suga N (1970) Echo-ranging neurons in the inferior colliculus of bats. Science 170:449-452.

Suga N, Schlegel P (1973) Coding and processing in the auditory systems of FM-signal-producing bats. J Acoust Soc Am 54:174-190.

Takahashi T, Moiseff A, Konishi M (1984) Time and intensity cues are processed independently in the auditory system of the owl. J Neurosci 4:1781-1786.

Tsuchitani C (1977) Functional organization of lateral cell groups of cat superior olivary complex. J Neurophysiol 40:296-318.

Tsuchitani C (1988a) The inhibition of cat lateral superior olive unit excitatory responses to binaural tone bursts. I. The transient chopper response. J Neurophysiol 59:164-183.

Tsuchitani C (1988b) The inhibition of cat lateral superior olive unit excitatory responses to binaural tone bursts. II. The sustained discharges. J Neurophysiol 59:184-211.

Warr WB (1966) Fiber degeneration following lesions in the anterior ventral cochlear nucleus of the cat. Exp Neurol 14:453-474.

Warr WB (1982) Parallel ascending pathways from the cochlear nucleus: neuroanatomical evidence of functional specialization. In: Contributions to sensory physiology, Vol 7 (Neff WD, ed), pp 1-38. New York: Academic.

Willard FH, Martin GF (1983) The auditory brainstem nuclei and some of their projections to the inferior colliculus in the North American opossum. Neuroscience 10:1203-1232.

Zook JM, Casseday JH (1982a) Cytoarchitecture of auditory system in lower brainstem of the mustache bat Pteronotus parnellii. J Comp Neurol 207:1-13.

Zook JM, Casseday JH (1982b) Origin of ascending projections to inferior colliculus in the mustache bat, Pteronotus parnellii. J Comp Neurol 207:14-28.

Zook JM, Casseday JH (1985) Projections from the cochlear nuclei in the mustache bat, Pteronotus parnellii. J Comp Neurol 237:307324.

Zook JM, Casseday JH (1987) Convergence of ascending pathways at the inferior colliculus in the mustache bat, Pteronotus parnellii. J Comp Neurol 261:347-361.

Zook JM, Jacobs MS, Glezer I, Morgane PJ (1988) Some comparative aspects of auditory brainstem cytoarchitecture in echolocating mammals: speculations on the morphological basis of time-domain processing. In: Animal sonar: processes and performance (Nachtigall PE, Moore PWB, eds), pp 311-316. New York: Plenum. 